\title{
Subarcsecond Observations of Ngc 7538 Irs 1: Continuum Distribution and Dynamics of Molecular Gas
}

\section{Citation}

Zhu, Lei, Jun-Hui Zhao, M. C. H. Wright, Göran Sandell, Hui Shi, Yue-Fang Wu, Crystal Brogan, and Stuartt Corder. 2013. "Subarcsecond Observations of Ngc 7538 Irs 1: Continuum Distribution and Dynamics of Molecular Gas." The Astrophysical Journal 779 (1): 51. https:// doi.org/10.1088/0004-637x/779/1/51.

\section{Permanent link}

http://nrs.harvard.edu/urn-3:HUL.InstRepos:41555825

\section{Terms of Use}

This article was downloaded from Harvard University's DASH repository, and is made available under the terms and conditions applicable to Other Posted Material, as set forth at http:// nrs.harvard.edu/urn-3:HUL.InstRepos:dash.current.terms-of-use\#LAA

\section{Share Your Story}

The Harvard community has made this article openly available.

Please share how this access benefits you. Submit a story.

\section{Accessibility}




\title{
SUBARCSECOND OBSERVATIONS OF NGC 7538 IRS 1: CONTINUUM DISTRIBUTION AND DYNAMICS OF MOLECULAR GAS
}

\author{
Lei Zhu ${ }^{1,2,3}$, Jun-Hui ZhaO ${ }^{2}$, M. C. H. Wright ${ }^{4}$, Göran SAndell ${ }^{5}$, Hui Shi ${ }^{1,6}$, Yue-Fang Wu ${ }^{3}$, \\ Crystal Brogan ${ }^{7}$, And Stuartt Corder $^{7}$ \\ ${ }^{1}$ National Astronomical Observatories, Chinese Academy of Sciences, A20 Datun Road, Chaoyang District, Beijing 100012, China; lzhu@nao.cas.cn \\ ${ }^{2}$ Harvard-Smithsonian Center for Astrophysics, 60 Garden Street, Cambridge, MA 02138, USA \\ ${ }^{3}$ Department of Astronomy, Peking University, Beijing 100871, China \\ ${ }^{4}$ Department of Astronomy, University of California, Berkeley, Berkeley, CA 94720, USA \\ ${ }^{5}$ SOFIA-USRA, NASA Ames Research Center, MS 232-12, Building N232, Rm. 146, P.O. Box 1, Moffett Field, CA 94035-0001, USA \\ ${ }^{6}$ Max-Planck Institut für Radioastronomie, Auf dem Hügel, 69, 53121, Bonn, Germany \\ ${ }^{7}$ NRAO, 520 Edgemont Road, Charlottesville, VA 22903, USA \\ Received 2013 February 3; accepted 2013 September 20; published 2013 November 25
}

\begin{abstract}
We report new results based on the analysis of the Submillimeter Array (SMA) and Combined Array for Research in Millimeter-wave Astronomy (CARMA) observations of NGC 7538 IRS 1 at 1.3 and $3.4 \mathrm{~mm}$ with subarcsecond resolutions. With angular resolutions $\sim 0^{\prime} 7$, the SMA and CARMA observations show that the continuum emission at 1.3 and $3.4 \mathrm{~mm}$ from the hyper-compact $\mathrm{H}$ II region IRS 1 is dominated by a compact source with a tail-like extended structure to the southwest of IRS 1 . With a CARMA B-array image at $1.3 \mathrm{~mm}$ convolved to 0.1 , we resolve the hyper-compact $\mathrm{H}$ II region into two components: an unresolved hyper-compact core, and a north-south extension with linear sizes of $<270 \mathrm{AU}$ and $\sim 2000 \mathrm{AU}$, respectively. The fine structure observed with CARMA is in good agreement with the previous Very Large Array results at centimeter wavelengths, suggesting that the hyper-compact $\mathrm{H}$ II region at the center of IRS 1 is associated with an ionized bipolar outflow. We image the molecular lines OCS(19-18) and $\mathrm{CH}_{3} \mathrm{CN}(12-11)$ as well as ${ }^{13} \mathrm{CO}(2-1)$ surrounding IRS 1, showing a velocity gradient along the southwest-northeast direction. The spectral line profiles in ${ }^{13} \mathrm{CO}(2-1), \mathrm{CO}(2-1)$, and $\mathrm{HCN}(1-0)$ observed toward IRS 1 show broad redshifted absorption, providing evidence for gas infall with rates in the range of 3-10 $\times 10^{-3} M_{\odot} \mathrm{yr}^{-1}$ inferred from our observations.
\end{abstract}

Key words: H II regions - ISM: jets and outflows - ISM: kinematics and dynamics - ISM: molecules - radio lines: ISM - stars: formation

Online-only material: color figures

\section{INTRODUCTION}

NGC 7538 IRS 1 is a hyper-compact (HC) $\mathrm{H}$ II region located at a distance of $2.65 \mathrm{kpc}$ (Moscadelli et al. 2009). Analysis of the radio and infrared (IR) data suggested that IRS 1 is an O6/7 star with a luminosity of a few times of $10^{5} L_{\odot}$ (Willner 1976; Akabane \& Kuno 2005). High-resolution observations of IRS 1 with the Very Large Array (VLA) showed two lobes of free-free emission within the central $2^{\prime \prime}$ region, located north and south of the emission peak of the HC H II region (Campbell 1984; Gaume et al. 1995; Sandell et al. 2009). The centimeter emission has been interpreted as a north-south ionized jet/outflow, while the large-scale outflow in $\mathrm{CO}$ appears to be along the northwest-southeast direction (e.g., Scoville et al. 1986; Kameya et al. 1989; Davis et al. 1998; Qiu et al. 2011). At shorter wavelengths, a northeast-southwest elongation of dust emission was observed in the mid-IR (De Buizer \& Minier 2005 ) and at $0.87 \mathrm{~mm}$, and several molecular lines show similar velocity gradients (Brogan et al. 2008; Klaassen et al. 2009; Beuther et al. 2012). In addition, a northwest-southeast linear structure of methanol masers with a velocity gradient of $\sim 0.02 \mathrm{~km} \mathrm{~s}^{-1} \mathrm{AU}^{-1}$ was found by Minier et al. $(1998,2000)$. The kinematics from the methanol maser emission was modeled as a circumstellar disk (Pestalozzi et al. 2004) with a major axis in the northwest-southeast direction, which is parallel to the axis of the large-scale outflow. Alternatively, the linear structure might be evidence that the methanol masers trace the inner wall of an outflow instead of a disk (Pestalozzi et al. 2004). To reconcile the various models from the previous observations in
$\mathrm{CO}$, methanol masers, radio continuum, and mid-IR emission, Kraus et al. (2006) proposed a precessing outflow model to interpret the observations.

In order to investigate the astrophysical process in the inner region of NGC 7538 IRS 1, we carried out high-angular resolution observations with the Submillimeter Array (SMA) ${ }^{8}$ at $1.3 \mathrm{~mm}$ and the Combined Array for Research in Millimeter-wave Astronomy (CARMA) at 1.3 and $3.4 \mathrm{~mm}$. SMA archival data were also used in our analysis of the molecular lines and continuum emission from IRS 1 . We present the results of the $1.3 \mathrm{~mm}$ and $3.4 \mathrm{~mm}$ continuum emission in Section 3 and molecular lines, including OCS(19-18), $\mathrm{CH}_{3} \mathrm{CN}(12-11), \mathrm{CO}(2-1),{ }^{13} \mathrm{CO}(2-1)$, and $\mathrm{HCN}(1-0)$, in Section 4 . The implications of the results are discussed in Section 5. Section 6 summarizes the conclusions.

\section{OBSERVATIONS AND DATA REDUCTIONS}

\subsection{SMA Data}

SMA observations of NGC 7538 IRS 1 in the very extended (VEX) configuration were made on 2008 August 5 with LO frequency $v_{\mathrm{LO}}=226.239 \mathrm{GHz}$, giving band-center frequencies of $221 \mathrm{GHz}$ for lower side band (LSB) and $231 \mathrm{GHz}$ for upper side band (USB). Eight antennas were used in the observations with the longest projected baseline $339 k \lambda$. The spectral resolution

\footnotetext{
8 The Submillimeter Array is a joint project between the Smithsonian Astrophysical Observatory and the Academia Sinica Institute of Astronomy and Astrophysics and is funded by the Smithsonian Institution and the Academia Sinica.
} 
Table 1

Log of Observation, $u v$ Data, and Images

\begin{tabular}{|c|c|c|c|c|c|c|}
\hline & & Visibility 1 & Sets & & & \\
\hline Obs. Date & & Pointing & Array Conf. & & Calibrator & \\
\hline & $\alpha_{\mathrm{J} 2000}$ & $\delta_{\mathrm{J} 2000}$ & & Phase & Bpass & Flux \\
\hline $\mathrm{SMA}$ at $1.3 \mathrm{~mm}$ & & & & & & \\
\hline 2008 May 5 & $23: 13: 45.362$ & $61: 28: 10.49$ & VEX & $\mathrm{J} 2202+422$ & $3 C 454.3$ & Vesta \\
\hline 2005 Sep 11 & $23: 13: 45.300$ & $61: 28: 10.00$ & EXT & $\mathrm{J} 0102+584$ & $3 C 454.3$ & Ceres \\
\hline & & & & $\mathrm{J} 2202+422$ & Neptune & \\
\hline CARMA at $1.3 \mathrm{~mm}$ & & & & & & \\
\hline 2010 Jan 4 & $23: 13: 45.600$ & $61: 28: 11.00$ & B-array & $\mathrm{J} 0102+584$ & $3 \mathrm{C} 454.3$ & Uranus, MWC349 \\
\hline 2010 Jan 5 & $23: 13: 45.600$ & $61: 28: 11.00$ & B-array & $\mathrm{J} 0102+584$ & $3 C 454.3$ & Neptune \\
\hline 2010 Nov 10 & $23: 13: 45.600$ & $61: 28: 11.00$ & C-array & $\mathrm{J} 0102+584$ & $3 C 454.3$ & Uranus \\
\hline 2009 Nov 23 & $23: 13: 45.600$ & $61: 28: 11.00$ & C-array & $\mathrm{J} 0102+584$ & 3C84 & Uranus \\
\hline 2009 Nov 27 & $23: 13: 45.600$ & $61: 28: 11.00$ & C-array & $\mathrm{J} 0102+584$ & $3 C 454.3$ & MWC349 \\
\hline 2010 Nov 29 & $23: 13: 45.600$ & $61: 28: 11.00$ & C-array & $\mathrm{J} 0102+584$ & $3 C 454.3$ & Neptune \\
\hline 2007 Mar 15 & $23: 13: 45.600$ & 61:28:11.00 & C-array & $\mathrm{J} 0102+584$ & $3 \mathrm{C} 454.3$ & MWC349 \\
\hline 2010 Aug 24 & $23: 13: 45.600$ & $61: 28: 11.00$ & D-array & $\mathrm{J} 0102+584$ & $3 C 454.3$ & Uranus \\
\hline 2009 Aug 7 & $23: 13: 45.600$ & $61: 28: 11.00$ & D-array & $\mathrm{J} 0102+584$ & $3 C 454.3$ & Neptune \\
\hline 2010 Aug 27 & $23: 13: 45.600$ & $61: 28: 11.00$ & D-array & $\mathrm{J} 0102+584$ & $3 C 454.3$ & Neptune \\
\hline 2010 Aug 27 & $23: 13: 45.600$ & $61: 28: 11.00$ & D-array & $\mathrm{J} 0102+584$ & $3 C 454.3$ & Neptune \\
\hline 2010 Sep 8 & $23: 13: 45.600$ & $61: 28: 11.00$ & D-array & $\mathrm{J} 0102+584$ & $3 C 454.3$ & Uranus \\
\hline 2010 Jul 5 & $23: 13: 45.600$ & $61: 28: 11.00$ & E-array & $\mathrm{J} 0102+584$ & $3 \mathrm{C} 454.3$ & MWC349 \\
\hline $2010 \mathrm{Jul} 7$ & $23: 13: 45.600$ & $61: 28: 11.00$ & E-array & $\mathrm{J} 0102+584$ & $3 C 454.3$ & MWC349 \\
\hline $2010 \mathrm{Jul} 8$ & $23: 13: 45.600$ & $61: 28: 11.00$ & E-array & $\mathrm{J} 0102+584$ & $3 C 454.3$ & Uranus \\
\hline CARMA at $3.4 \mathrm{~mm}$ & & & & & & \\
\hline 2010 Dec 29 & $23: 13: 45.298$ & $61: 28: 10.00$ & B-array & $\mathrm{J} 0102+584$ & $3 C 454.3$ & Uranus \\
\hline & & Continuum anc & Images & & & \\
\hline Mode & $\begin{array}{l}\text { FWHM Beam } \\
\text { " } \times "\left({ }^{\circ}\right)\end{array}$ & $\begin{array}{c}\text { rms } \\
\left(\mathrm{mJy} \mathrm{beam}^{-1}\left(\text { channel }^{-1}\right)\right)\end{array}$ & $\begin{array}{c}\mathrm{BW} \\
(\mathrm{GHz})\end{array}$ & $\begin{array}{c}\delta V \\
\left(\mathrm{~km} \mathrm{~s}^{-1}\right)\end{array}$ & $\begin{array}{l}V_{\mathrm{LSR}} \text {-ranges } \\
\left(\mathrm{km} \mathrm{s}^{-1}\right)\end{array}$ & $u v$ Data Sets \\
\hline SMA & & & & & & \\
\hline Continuum $1.3 \mathrm{~mm}$ & $0.8 \times 0.6(-45)$ & 4 & 4 & $\ldots$ & $\ldots$ & $\mathrm{VEX}+\mathrm{EXT}$ \\
\hline Line OCS(19-18) & $0.8 \times 0.6(-45)$ & 60 & $\ldots$ & 0.5 & -85 to -35 & $\mathrm{VEX}+\mathrm{EXT}$ \\
\hline Lines $\mathrm{CH}_{3} \mathrm{CN}(12-11)$ & $0.8 \times 0.6(-45)$ & 60 & $\ldots$ & 0.5 & -85 to -35 & VEX + EXT \\
\hline Line ${ }^{13} \mathrm{CO}(2-1)$ & $0.8 \times 0.6(-45)$ & 50 & $\ldots$ & 0.5 & -110 to -10 & $\mathrm{VEX}+\mathrm{EXT}$ \\
\hline Line ${ }^{12} \mathrm{CO}(2-1)$ & $0.8 \times 0.6(-45)$ & 60 & $\ldots$ & 1 & -160 to +40 & $\mathrm{VEX}+\mathrm{EXT}$ \\
\hline CARMA & & & & & & \\
\hline Continuum $1.3 \mathrm{~mm}$ & $0.78 \times 0.64(-84)$ & 2.5 & $2-3$ & $\ldots$ & $\ldots$ & $\mathrm{B}+\mathrm{C}+\mathrm{D}+\mathrm{E}$-array \\
\hline Continuum $1.3 \mathrm{~mm}$ & $0.34 \times 0.27(-83)$ & 10 & 2 & $\ldots$ & $\ldots$ & B-array \\
\hline Continuum $3.4 \mathrm{~mm}$ & $0.8 \times 0.7(-68)$ & 7 & 0.064 & $\ldots$ & $\ldots$ & B-array \\
\hline Line $\mathrm{HCN}(1-0)$ & $0.8 \times 0.7(-68)$ & 33 & $\ldots$ & 1.65 & -220 to -11 & B-array \\
\hline
\end{tabular}

was $0.81 \mathrm{MHz}$, which corresponds to a velocity resolution of $\sim 1.1 \mathrm{~km} \mathrm{~s}^{-1}$. The total on-source integration time was $82 \mathrm{~min}$ utes. The average system temperature was $180 \mathrm{~K}$.

NGC 7538 IRS 1 was also observed with the SMA in the extended (EXT) configuration on 2005 September 11, with an LO frequency $\nu_{\text {LO }}=226.348 \mathrm{GHz}$. Six of the eight SMA antennas were used, giving the longest projected baseline $133 \mathrm{k} \lambda$. A standard correlator configuration was used with a spectral resolution of $0.33 \mathrm{MHz}$, corresponding to a velocity resolution of $0.42 \mathrm{~km} \mathrm{~s}^{-1}$. The total on-source integration time was 309 minutes, and the average system temperature was $105 \mathrm{~K}$

The data reduction and analysis were made in MIRIAD (Sault et al. 1995) following the reduction instructions for SMA data. ${ }^{9}$ For the EXT array data set (2005), system temperature corrections were applied to remove the atmospheric attenuation. Antenna-based bandpass solutions were calculated over three time intervals (UT 4:45-5:40 for Neptune, and UT 6:00-6:30 and UT 14:00-14:45 for 3C454.3) to interpolate the corrections

\footnotetext{
9 http://www.cfa.harvard.edu/sma/miriad
}

for the bandpass ripples due to instrument defects. After that, baseline-based and spectral-window based residual errors in the bandpass were further corrected using the point source 3C454.3. The time-dependent gains were derived and interpolated from two nearby QSOs, J0102+584 and J2202+422; the complex gain corrections were applied to the target source. Finally, the flux-density scale was bootstrapped from Ceres using the SMA planetary model assuming a flux density of $0.95 \mathrm{Jy}$ and an angular size of 0!'43. The reduction of the VEX array data is similar, and the calibrators used in the data reduction for the EXT and VEX configuration data are summarized in Table 1.

In order to image the continuum emission and the molecular lines in the IRS 1 region with high-angular resolution and good $u v$ coverage, we combined the EXT (2005) and VEX (2008) data sets. The EXT and VEX visibility data sets have an offset of 0 '.7 in pointing position. Using UVEDIT in MIRIAD, we shifted the two data sets to a common phase reference center at the continuum peak position determined from the EXT (2005) data set. Then, the residual phase errors in the continuum were further corrected using the self-calibration technique with a point-source model at the position of the radio compact source 
in IRS 1, which was determined from the VLA image at $43 \mathrm{GHz}$ (Sandell et al. 2009). The self-calibration solutions determined from continuum data were also applied to the line data sets.

The continuum data sets were created by averaging all the line-free channel data using UVLIN. By combining the USB and LSB data sets of the EXT and VEX array configurations, the continuum image at $1.3 \mathrm{~mm}$ was constructed. The continuumfree line visibility data sets were created by subtracting the continuum level, which was determined by a linear interpolation of the line-free channels using UVLIN in MIRIAD.

In addition to the $1.3 \mathrm{~mm}$ results, we also included the SMA data of NGC 7538 IRS 1 at $345 \mathrm{GHz}$ which were observed in 2005 (Brogan et al. 2008). The FWHM beam sizes for the final line images are about $2^{\prime \prime}$. We analyzed the relatively optically thin lines of species $\mathrm{CH}_{3} \mathrm{OH}$ and ${ }^{13} \mathrm{CH}_{3} \mathrm{OH}$ to determine the systemic velocity of the IRS 1 system.

\subsection{CARMA Data}

$$
\text { 2.2.1. } 1.3 \mathrm{~mm}
$$

High-resolution observations of IRS 1 were carried out on 2010 January 4 and 5 with CARMA in the B array configuration at $1.3 \mathrm{~mm}$. The CARMA observations used two $500 \mathrm{MHz}$ bands in USB and LSB of LO1 with a total continuum bandwidth of $2 \mathrm{GHz}$. The data were reduced and imaged in a standard way using MIRIAD software. The quasar J0102+584 was used as a gain and phase calibrator, and Uranus and MWC 349 were used for flux and bandpass calibration. The uncertainty in the absolute flux-density scale was $\sim 20 \%$. The strong compact emission from IRS 1 was used to self-calibrate IRS 1 with respect to the $43 \mathrm{GHz}$ VLA image with a Gaussian fit position R.A. $(\mathrm{J} 2000)=23^{\mathrm{h}} 13^{\mathrm{m}} 45^{\mathrm{s}} \cdot 37$, decl. $(\mathrm{J} 2000)=61^{\circ} 28^{\prime} 10^{\prime \prime} .4$. The continuum image was constructed by combining the two$500 \mathrm{MHz}$ sidebands in a multi-frequency synthesis (MFS) mosaic. The CARMA data with three different primary beams resulting from the 6.1 and $10.4 \mathrm{~m}$ antenna pairs were used in the mosaic imaging. The weighted mean observing frequency from MFS is $222.2 \mathrm{GHz}$.

To verify the structure observed with the SMA, we used the lower-resolution data from the CARMA observations in the $\mathrm{C}, \mathrm{D}$, and $\mathrm{E}$ array configurations that were carried out between 2007 and 2010. The setup of the corresponding observations is summarized in Table 1, giving the observing date, pointing positions, band information, array configurations, and calibrators of these observations. All these observations were made with two or three $500 \mathrm{MHz}$ wide bands in USB and LSB with total bandwidths of 2 or $3 \mathrm{GHz}$. The continuum data were calibrated following the standard procedure. The calibrated data sets were split into single source data sets for the target source IRS 1. With UVEDIT, the phase-centers of each data set were shifted to the common position of the IRS 1 continuum source determined from the VLA image at $43 \mathrm{GHz}$. Then, the residual complex gain errors in the $\mathrm{B}, \mathrm{C}, \mathrm{D}$, and $\mathrm{E}$ array data sets were corrected using self-calibration with an initial model of a point source at the phase center. The structure seen in the B array image, which was self-calibrated with the model from the $43 \mathrm{GHz}$ VLA image (as discussed above), was confirmed from this self-calibration procedure using a point source as an initial model.

$$
\text { 2.2.2. } 3.4 \mathrm{~mm}
$$

We also carried out observations at $89 \mathrm{GHz}$ with the CARMA telescope in the B array configuration with 15 antennas on 2010
December 28. We used a correlator setup with 16 spectral windows (including USB and LSB), four with $500 \mathrm{MHz}$ bandwidth (39 channels in total and $12.5 \mathrm{MHz}$ for each channel), four with $250 \mathrm{MHz}$ (79 channels in total and $3.225 \mathrm{MHz}$ for each channel), and the remaining eight windows with $64 \mathrm{MHz}$ bandwidth (255 channels in total and $0.244 \mathrm{MHz}$ for each channel).

The data were calibrated and imaged following the standard procedure for CARMA data in MIRIAD. The HCN(1-0) line at $v_{0}=88.6318 \mathrm{GHz}$ was included in one of the narrow-band windows. The continuum data set was made from the linefree channels in this window. The residual phase errors were corrected using the self-calibration technique. The position of the IRS 1 continuum source at $3.4 \mathrm{~mm}$ was offset $\left(-00^{\prime} .18,-00^{\prime} .10\right)$ from the SMA position at $1.3 \mathrm{~mm}$, which is within the positional uncertainty in the SMA image. We binned two channels together to make a line image of $\mathrm{HCN}(1-0)$ with the same FWHM beam size as the continuum image.

A log summarizing the observations, $u v$ data sets, and images is given in Table 1 .

\section{STRUCTURE OF CONTINUUM EMISSION FROM IRS 1}

With an angular resolution of $\sim 0^{\prime} .7$, both the SMA (Figure 1(a)) and CARMA (Figure 1(b)) show extended emission with a peak flux density of $0.12 \pm 0.02 \mathrm{Jy} \mathrm{beam}^{-1}$, located $\sim 1^{\prime \prime}$ SW of the unresolved component in IRS 1. Lacking centimeter counterparts, the extended continuum emission is likely to correspond to thermal dust emission, which could contain additional dust cores in the IRS 1 region. It is also plausible that the SW extension corresponds to a dust component that is located along the interface between the ionized and molecular medium and is compressed by the ionized outflow.

The CARMA image at $3.4 \mathrm{~mm}$ (Figure 1(c)) shows a pointlike source with no significant detection of the SW extension. A total flux density of $0.93 \pm 0.10 \mathrm{Jy}$ was determined for the central unresolved core. Assuming a flat power-law $(\beta \sim 0.5$ for dust emissivity), a peak flux density of $0.01 \mathrm{Jy}^{\text {beam }}{ }^{-1}$ is extrapolated from the $1.3 \mathrm{~mm}$ peak flux density of the SW extension, which is below the $3 \sigma$ limit of the $3.4 \mathrm{~mm}$-image.

The CARMA $1.3 \mathrm{~mm}$ image (Figure 1(d)), convolved to a circular beam of 0 .' 1 , shows a central emission peak with a flux density of $1.0 \pm 0.1 \mathrm{Jy}$ beam $^{-1}$. The innermost part of the HC $\mathrm{H}$ II region at $1.3 \mathrm{~mm}$ is unresolved, this could be the optically thick part of the ionized outflow containing an accretion disk in IRS 1 . Because the optically thick free-free emission dominates the $1.3 \mathrm{~mm}$ continuum, as well as the limitation of the angular resolution, the postulated ionized disk in IRS 1 has not been detected. In the CARMA $1.3 \mathrm{~mm}$ image (Figure 1(d)) extensions along the south and north directions from the bright point source have been revealed with a scale of 0.8 above a noise level of $4 \sigma$. The emitting lobes appear to be from the inner portion of the ionized outflow.

Previous studies show that the continuum emission from IRS 1 is dominated by the free-free emission from a bipolar ionized outflow at frequencies lower than $300 \mathrm{GHz}$ (Sandell et al. 2009). Figure 2 compares the CARMA $1.3 \mathrm{~mm}$ image with previous VLA images. At wavelengths $\lambda \geqslant 1.3 \mathrm{~cm}$, a dark lane divides the two emission peaks in the center of the IRS 1 region, which is probably due to the self-absorption in the ionized gas. At shorter wavelengths, $\lambda \leqslant 7 \mathrm{~mm}$, a single emission peak appears, located at R.A. $(\mathrm{J} 2000)=23^{\mathrm{h}} 13^{\mathrm{m}} 45^{\mathrm{s}} .37$, decl. $(\mathrm{J} 2000)=61^{\circ} 28^{\prime} 10^{\prime} .43$. Hereafter, we take this position as the reference to register both the SMA and CARMA images. 

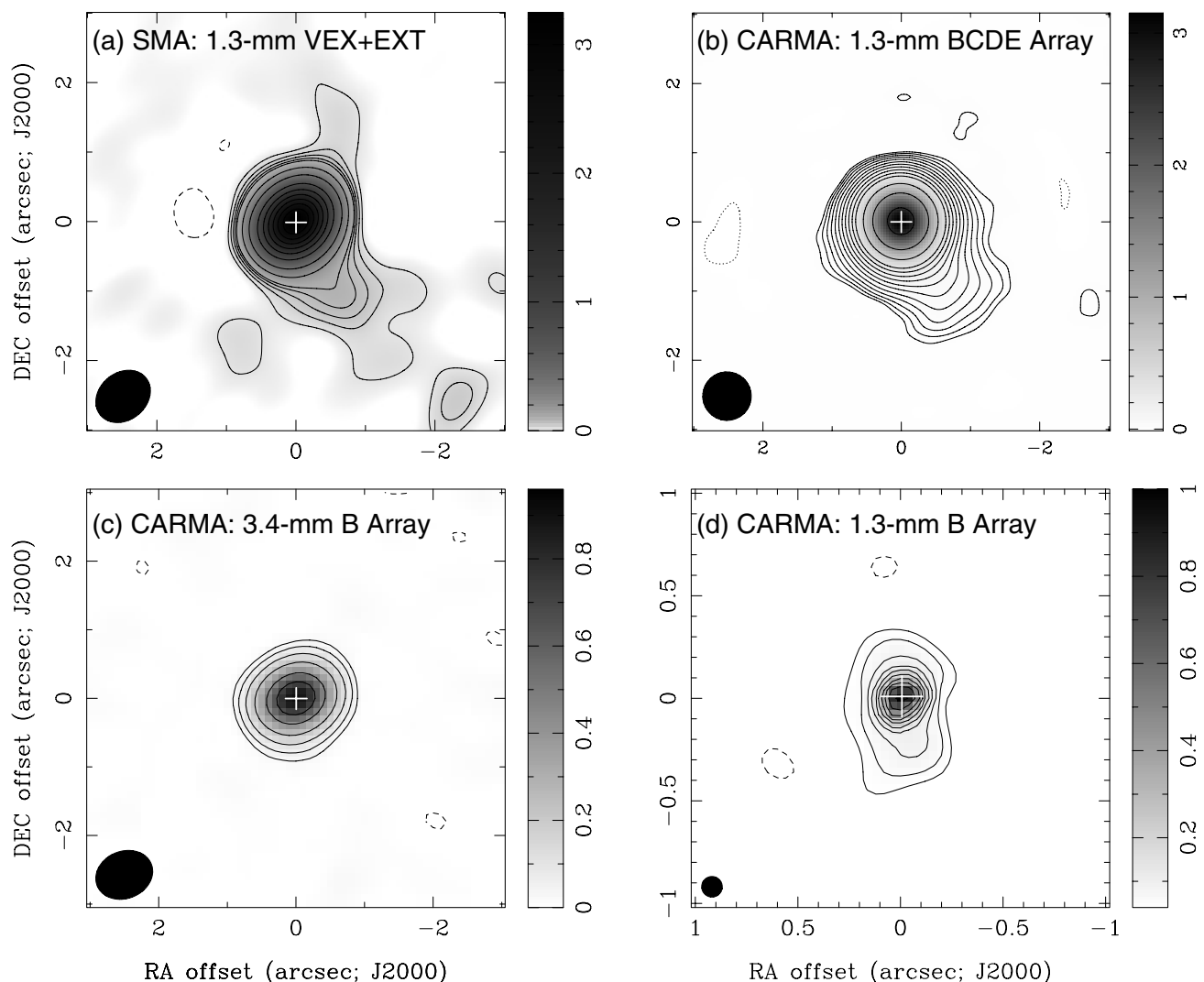

Figure 1. (a) The SMA $1.3 \mathrm{~mm}$ continuum image made with the line-free channels by combining LSB and USB data from both VEX and EXT array observations. The contours are $\left(-5,5,7,10,14,20,28,40,56,80,112,160,226,320,452,640,905\right.$, and 1280) $\times \sigma$ and $\sigma=4$ mJy beam $^{-1}$. The FWHM beam size is $00^{\prime \prime} 8 \times 00^{\prime \prime} 6$, P.A. $=-45^{\circ}$. (b) The CARMA $1.3 \mathrm{~mm}$ continuum image with lower-resolution made by combining B, C, D, and E array configuration data, convolved to a circular beam (FWHM) of $0 !$ ' 7 , with rms noise $\sigma=2.5 \mathrm{mJy}$ beam $^{-1}$. The contours are $(-4,4,5.7,8,11.3,16,23,32,45,64,91,128,256,512$, and 1028$) \times \sigma$. (c) The CARMA $3.4 \mathrm{~mm}$ continuum image made from the CARMA B-array observations with FWHM beam of $0^{\prime \prime} 8 \times 00^{\prime \prime} 7, \mathrm{P} . \mathrm{A} .=-68^{\circ}$. The contours are $(-2,4,8,16,32,64$, and 96) $\times \sigma$. The rms noise $\sigma=7 \mathrm{mJy}_{\text {beam }}{ }^{-1}$. In all the images the reference position is R.A. (J2000) $=23^{\mathrm{h}} 13^{\mathrm{m}} 45^{\mathrm{s}} \cdot 37$, decl. $(\mathrm{J} 2000)=61^{\circ} 28^{\prime} 10^{\prime \prime} 43$, which is the peak position of $43 \mathrm{GHz}$ continuum emission and marked by a plus. The FWHM of the synthesized beam is indicated at the left-bottom corner of each image. The grayscale of the wedges is in the units of Jy beam ${ }^{-1}$. (d) The CARMA $1.3 \mathrm{~mm}$ continuum image of the central region of IRS 1 with a higher-resolution made with the B-array data only, convolved to a circular beam (FWHM) of $0^{\prime \prime} 1$, and with rms noise $\sigma=10$ mJy beam ${ }^{-1}$. The contours are $(-4,4,5.7,8,11.3,16,23,32,45,64$, and 91$) \times \sigma$.

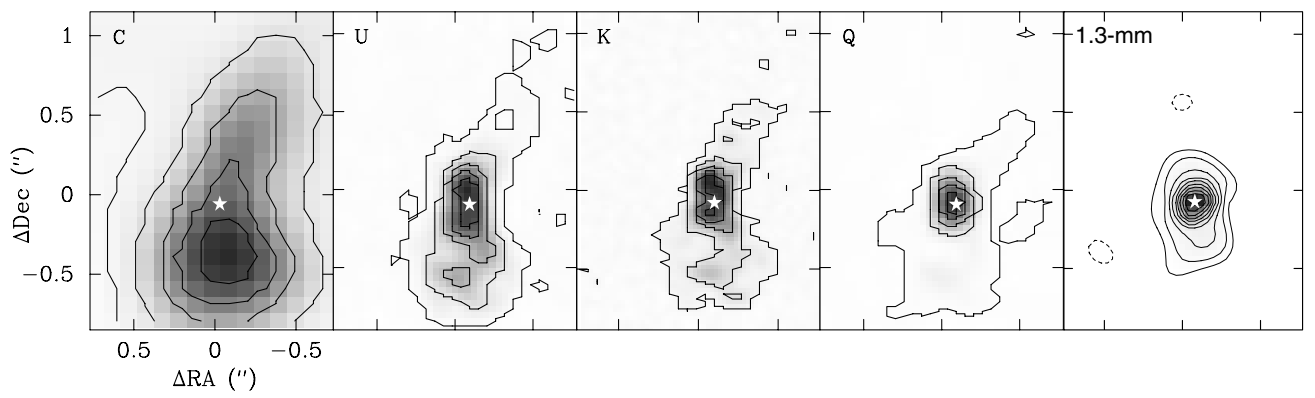

Figure 2. CARMA high-resolution image of IRS 1 at $1.3 \mathrm{~mm}$ is compared with the images observed with the VLA at $6,2.0,1.3$, and $0.7 \mathrm{~cm}$ (Sandell et al 2009). Different from other figures in this paper, this reference center is the same as the original figure in Sandell et al. (2009). The white stars mark the position R.A. $(\mathrm{J} 2000)=23^{\mathrm{h}} 13^{\mathrm{m}} 45^{\mathrm{s}} \cdot 37$, decl. $(\mathrm{J} 2000)=61^{\circ} 28^{\prime} 10^{\prime \prime} 43$.

The properties of the continuum sources in the IRS 1 region are summarized in Table 2 .

\section{MOLECULAR LINES}

\subsection{Systemic Velocity}

At the $1.3 \mathrm{~mm}$ wavelength, the SMA data for NGC 7538 IRS 1 show numerous strong molecular emission lines along with a few absorption features. A large portion of the emission is from the molecular lines with high excitation energy levels (e.g., OCS, $\mathrm{CH}_{3} \mathrm{CN}, \mathrm{CH}_{3} \mathrm{OCH}_{3}$, and $\mathrm{C}_{2} \mathrm{H}_{5} \mathrm{OH}$ ), showing a strong emission peak at a radial velocity of $-59.5 \mathrm{~km} \mathrm{~s}^{-1}$ with a scatter of about one channel width $\left(\sim 0.5 \mathrm{~km} \mathrm{~s}^{-1}\right)$. This value is close to the velocity $\left(-59.7 \pm 0.3 \mathrm{~km} \mathrm{~s}^{-1}\right)$ observed in the mid-IR by Knez et al. (2009). In addition, we further investigated the $0.86 \mathrm{~mm}$ SMA data and analyzed the $\mathrm{CH}_{3} \mathrm{OH}$ $(12,1,11-12,0,12),{ }^{13} \mathrm{CH}_{3} \mathrm{OH}(12,1,11-12,0,12)$, and ${ }^{13} \mathrm{CH}_{3} \mathrm{OH}$ $(14,1,13-14,0,14)$ lines. The integrated intensity images and line profiles are shown in Figure 3, and the Gaussian fitting to the line profiles are summarized in Table 3. The Gaussian fitting shows an average peak velocity of $-58.6 \pm 0.2 \mathrm{~km} \mathrm{~s}^{-1}$ for these lines, which have higher excitation energy than the ones observed in the $1.3 \mathrm{~mm}$ SMA data, as well as lower optical depths (for the two rare isotopic ones). On the other hand, because the $\sim 2^{\prime \prime}$ 

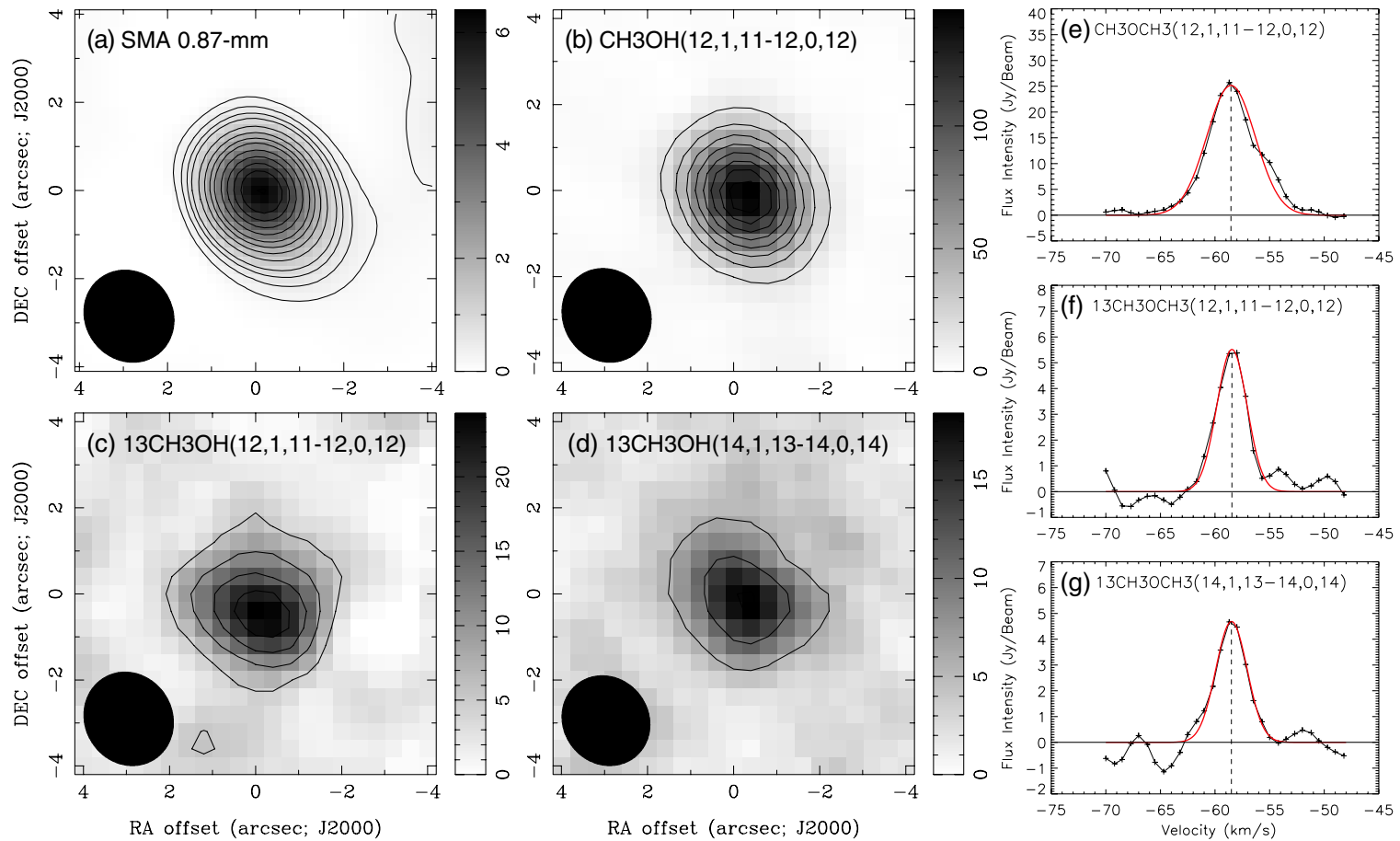

Figure 3. (a) The SMA $0.87 \mathrm{~mm}$ continuum image with a FWHM beam size of $2^{\prime \prime} 1 \times 22^{\prime \prime} 0$, P.A. $=38^{\circ}$. The contours are $(-1,1,2,3,4,6,8,10,12,15,18,21,24$, and 28) $\times 5 \sigma$, and the rms noise is $\sigma=45 \mathrm{mJy}_{\text {beam }}{ }^{-1}$. (b) The integrated intensity map of the line $\mathrm{CH}_{3} \mathrm{OH}(12,1,11-12,0,12)$ with a FWHM beam size of 2 '. $2 \times$ $2^{\prime \prime} .0$, P.A. $=29^{\circ}$. The contours are $(-1,1,2,3,4,5,6,7$, and 8$) \times 10 \sigma$, and the rms noise is $\sigma=1.7 \mathrm{Jy} \mathrm{beam}^{-1} \mathrm{~km} \mathrm{~s}^{-1}$. (c) The integrated intensity map of the line ${ }^{13} \mathrm{CH}_{3} \mathrm{OH}(12,1,11-12,0,12)$ with a FWHM beam size of $2^{\prime \prime} .2 \times 22^{\prime \prime} 0$, P.A. $=31^{\circ}$. The contours are $(-1,1,2,3,4,5,6,7$, and 8$) \times 3 \sigma$, and the rms noise is $\sigma=1.7 \mathrm{Jy} \mathrm{beam}^{-1} \mathrm{~km} \mathrm{~s}^{-1}$. (d) The integrated intensity map of the line ${ }^{13} \mathrm{CH}_{3} \mathrm{OH}(14,1,13-14,0,14)$ with a FWHM beam size of $2^{\prime \prime} \cdot 1 \times 2^{\prime \prime} \cdot 0$, P.A. $=38^{\circ}$. The contours are $(-1,1,2,3,4,5,6,7$, and 8$) \times 3 \sigma$, and the rms noise is $\sigma=2.0 \mathrm{Jy} \mathrm{beam}^{-1} \mathrm{~km} \mathrm{~s}^{-1}$. (e) $-(\mathrm{g})$ The spectra of $\mathrm{CH}_{3} \mathrm{OH}(12,1,11-12,0,12),{ }^{13} \mathrm{CH}_{3} \mathrm{OH}(12,1,11-12,0,12)$, and ${ }^{13} \mathrm{CH}_{3} \mathrm{OH}(14,1,13-14,0,14)$ at the peak position of the continuum emission. The red lines in (e)-(g) are the results of Gaussian fitting for the line profiles.

(A color version of this figure is available in the online journal.)

Table 2

Results from Continuum Observations at 1.3 and $3.4 \mathrm{~mm}$

\begin{tabular}{|c|c|c|c|c|}
\hline Source & $\begin{array}{c}S_{\mathrm{p}} \\
\left(\mathrm{Jy} \mathrm{beam}^{-1}\right)\end{array}$ & $\begin{array}{c}S_{\mathrm{t}} \\
(\mathrm{Jy})\end{array}$ & $\begin{array}{c}\Delta \alpha, \Delta \delta \\
\left({ }^{\prime \prime},{ }^{\prime \prime}\right)\end{array}$ & $\begin{array}{c}\Delta \theta_{\mathrm{M}} \times \Delta \theta_{\mathrm{m}}(\text { P.A. }) \\
\left({ }^{\prime \prime} \times^{\prime \prime}\left({ }^{\circ}\right)\right)\end{array}$ \\
\hline \multicolumn{5}{|l|}{ SMA results } \\
\hline \multicolumn{5}{|l|}{ SMA $1.3 \mathrm{~mm}$} \\
\hline IRS 1 & $3.3 \pm 0.3$ & $3.5 \pm 0.4$ & $0.0 \pm 0.1,0.0 \pm 0.1$ & Unresolv \\
\hline SW extension & $0.12 \pm 0.02$ & $0.25 \pm 0.04$ & $-0.5 \pm 0.1,-0.9 \pm 0.1$ & $1.0 \times 0.6(22)$ \\
\hline \multicolumn{5}{|l|}{ CARMA $1.3 \mathrm{~mm}$} \\
\hline IRS 1-peak & $1.0 \pm 0.1$ & $1.0 \pm 0.1$ & $-0.08 \pm 0.02,-0.07 \pm 0.02$ & Unresolv \\
\hline IRS 1-lobe & $0.22 \pm 0.02$ & $2.8 \pm 0.3$ & $-0.08 \pm 0.01,-0.09 \pm 0.01$ & $0.42 \times 0.28(-2)$ \\
\hline IRS 1-total & $\ldots$ & $3.8 \pm 0.4$ & $\ldots$ & $\ldots$ \\
\hline \multicolumn{5}{|l|}{ CARMA $3.4 \mathrm{~mm}$} \\
\hline IRS 1 & $0.93 \pm 0.10$ & $0.93 \pm 0.10$ & $0.0 \pm 0.1,0.0 \pm 0.1$ & Unresolv \\
\hline
\end{tabular}

Note. The reference position is R.A. $(\mathrm{J} 2000)=23^{\mathrm{h}} 13^{\mathrm{m}} 45^{\mathrm{s}} \cdot 37$, decl. $(\mathrm{J} 2000)=61^{\circ} 28^{\prime} 10^{\prime \prime} 43$.

Table 3

Fitting of the High Transition Lines at $0.86 \mathrm{~mm}$

\begin{tabular}{lcccc}
\hline \hline Transition & $\begin{array}{c}v \\
(\mathrm{GHz})\end{array}$ & $\begin{array}{c}\Delta S_{\mathrm{L}} \\
\left(\mathrm{Jy} \mathrm{beam}^{-1}\right)\end{array}$ & $\begin{array}{c}V_{\text {LSR }} \\
\left(\mathrm{km} \mathrm{s}^{-1}\right)\end{array}$ & $\begin{array}{c}\Delta V_{\text {FWHM }} \\
\left(\mathrm{km} \mathrm{s}^{-1}\right)\end{array}$ \\
\hline $\mathrm{CH}_{3} \mathrm{OH}(12,1,11-12,0,12)$ & 336.865 & $27.8 \pm 0.4$ & $-58.7 \pm 0.1$ & $4.8 \pm 0.2$ \\
${ }^{13} \mathrm{CH}_{3} \mathrm{OH}(12,1,11-12,0,12)$ & 335.560 & $6.2 \pm 0.2$ & $-58.5 \pm 0.1$ & $3.1 \pm 0.2$ \\
${ }^{13} \mathrm{CH}_{3} \mathrm{OH}(14,1,13-14,0,14)$ & 347.188 & $4.9 \pm 0.2$ & $-58.6 \pm 0.1$ & $3.4 \pm 0.2$ \\
\hline
\end{tabular}

angular resolution of the $0.86 \mathrm{~mm}$ observations is poor compared to the $1.3 \mathrm{~mm}$ observations, it is possible that the $0.86 \mathrm{~mm}$ emission includes the kinematics of surrounding medium (e.g., outflow or infall). Therefore, considering the results of all the observations, we adopt $V_{\text {sys }}=-59.0 \pm 0.5 \mathrm{~km} \mathrm{~s}^{-1}$ as the systemic velocity of the IRS 1 system in the following analysis.

\subsection{Line Identifications of the $1.3 \mathrm{~mm}$ SMA Data}

Figure 4 shows the spectra from the $1.3 \mathrm{~mm}$ SMA data observed in the EXT configuration, which are corrected for the systemic velocity, $V_{\text {sys }}=-59.0 \mathrm{~km} \mathrm{~s}^{-1}$. Numerous molecular lines were identified on the basis of the molecular line surveys toward Sgr B2 (Nummelin et al. 1998) and Orion (Sutton et al. 1985), as well as other available line catalogs. The names of the identified molecules are labeled in Figure 4.

With no significant blending with other lines, OCS(19-18) and multiple $K$ transitions of $\mathrm{CH}_{3} \mathrm{CN}(12-11)$ included in both 

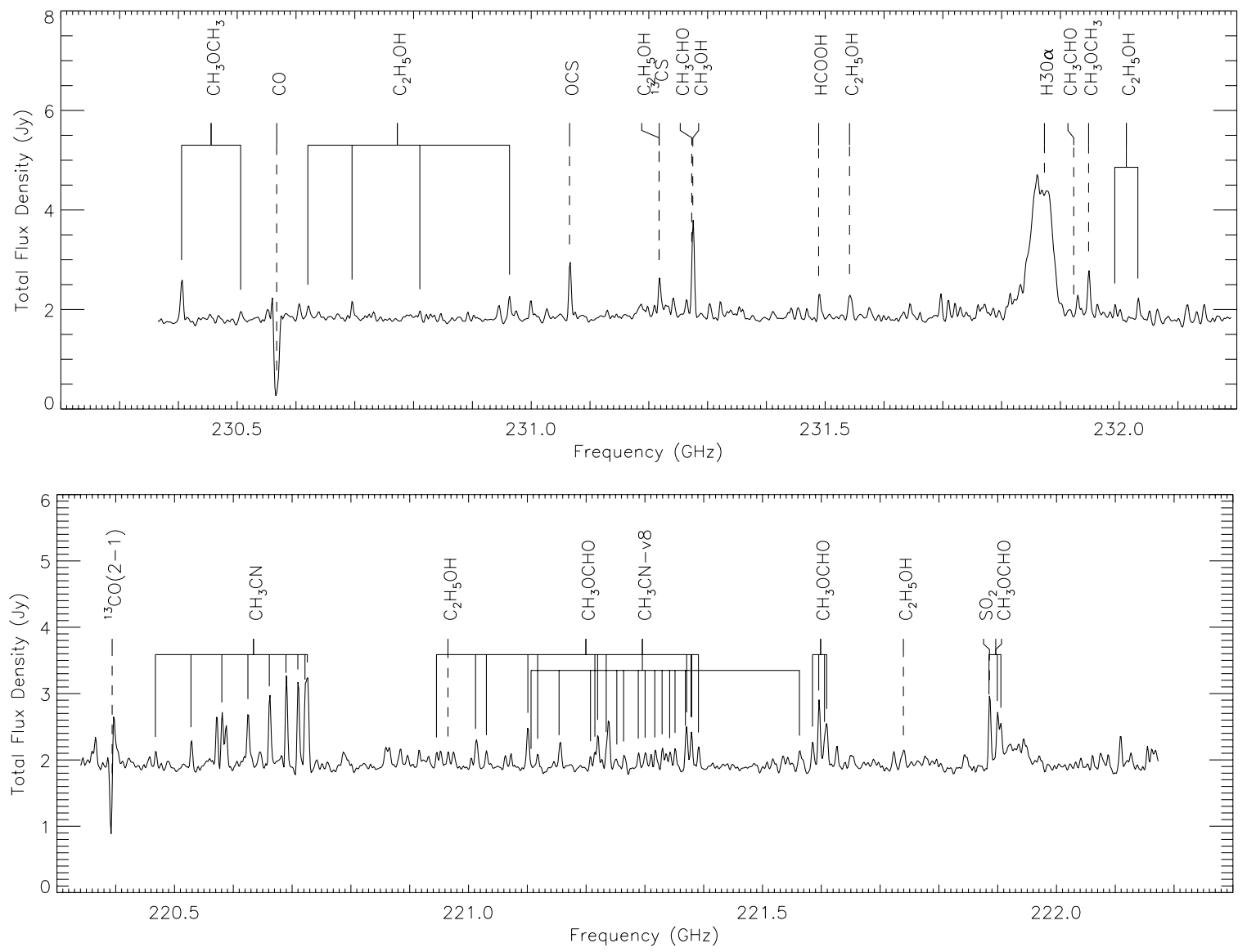

Figure 4. Spectra toward IRS 1 that were made from the upper (top) and lower (bottom) side bands based on the observations with the SMA on 2005 September 11 in the extended array configuration. The spectra have been converted to the rest frame at $V_{\mathrm{sys}}=-59.0 \mathrm{~km} \mathrm{~s}^{-1}$. The identifications for the major molecular and hydrogen recombination lines are labeled.

EXT and VEX configuration observations were selected in our analysis to determine the excitation condition and kinematics in the IRS 1 region.

\section{3. $O C S(19-18)$}

The channel maps of the OCS(19-18) line show two significant velocity components in the OCS(19-18) emission (see Figure 5). The main OCS(19-18) emission component in the velocity range from -62.5 to $-57.0 \mathrm{~km} \mathrm{~s}^{-1}$ is referred to as IRS $1 \mathrm{SW}$ as it is located to the south and southwest of the $\mathrm{HC}$ H II region IRS 1, while the fainter, less extended redshifted component, hereafter IRS $1 \mathrm{NE}$, is located to the northeast of IRS 1 ranging from -52.0 to $-47.0 \mathrm{~km} \mathrm{~s}^{-1}$. No significant OCS(19-18) emission is present in the velocity range between -56.5 and $-53.0 \mathrm{~km} \mathrm{~s}^{-1}$. This velocity gap in emission is redshifted with respect to $V_{\text {sys }}=-59.0 \mathrm{~km} \mathrm{~s}^{-1}$.

Figure 6(a) shows the integrated line intensity of the two components SW and NE. We fitted the two components with two-dimensional Gaussians as well as their line profiles with multiple Gaussians. The fitting results are summarized in Table 4 . The peak position of IRS $1 \mathrm{SW}$ is offset $\left(-0^{\prime \prime} .13 \pm 0^{\prime \prime} 03\right.$, $-0.41 \pm 0$ '03) with respect to IRS 1 , while the offset of IRS 1 $\mathrm{NE}$ is $\left(00^{\prime} 25 \pm 00^{\prime} 03,00^{\prime} 35 \pm 00^{\prime} \cdot 02\right)$. The total integrated line flux of OCS(19-18) for IRS $1 \mathrm{SW}\left(10.1 \pm 0.7 \mathrm{Jy} \mathrm{km} \mathrm{s}^{-1}\right)$ is about three times the value for IRS $1 \mathrm{NE}\left(3.8 \pm 0.6 \mathrm{Jy} \mathrm{km} \mathrm{s}^{-1}\right)$.

Figure 6(b) shows the line profiles of the OCS(19-18) spectra at the peak positions of IRS $1 \mathrm{SW}$ and NE, and Figure 6(c) shows the spectral profile integrated over the entire IRS 1 region. Gaussian fits to the observed spectral profiles give a radial velocity $V_{\mathrm{LSR}}=-59.5 \pm 0.1 \mathrm{~km} \mathrm{~s}^{-1}$ with a line width $\Delta V_{\mathrm{FWHM}}=4.4 \pm 0.1 \mathrm{~km} \mathrm{~s}^{-1}$ for IRS $1 \mathrm{SW}$, and $V_{\mathrm{LSR}}=-51.5 \pm 0.3 \mathrm{~km} \mathrm{~s}^{-1}$ and $\Delta V_{\mathrm{FWHM}}=7.2 \pm 0.6 \mathrm{~km} \mathrm{~s}^{-1}$ for the weaker emission feature (IRS $1 \mathrm{NE}$; see Table 4). The centroid velocity of IRS $1 \mathrm{SW}$ is close to $V_{\text {sys }}=-59.0 \mathrm{~km} \mathrm{~s}^{-1}$, which is used in this paper, suggesting that ambient gas might dominate this component. The spectrum of IRS $1 \mathrm{NE}$ is not a well-defined Gaussian shape, and the large line width suggests that it is subject to more dynamic interaction than IRS 1 SW.

\section{4. $\mathrm{CH}_{3} \mathrm{CN}(12-11)$ Lines}

The $\mathrm{CH}_{3} \mathrm{CN}(12-11) K$-ladder with $K=0-8$, which covers the frequencies ranging from $v_{K=8}(220.476 \mathrm{GHz})$ to $v_{K=0}$ $(220.747 \mathrm{GHz})$, were all detected in IRS 1 (see Figure 7). Similar to what is seen in OCS(19-18), the $\mathrm{CH}_{3} \mathrm{CN}(12-11)$ lines also show an emission peak at $\sim-59.5 \mathrm{~km} \mathrm{~s}^{-1}$ with weaker redshifted components. At the intensity peak of IRS $1 \mathrm{SW}$, the line profiles of $K=0-8$ transitions are shown in Figures 7(a) and (b), while as for IRS $1 \mathrm{NE}$, only $K=0-7$ transitions are detected above $3 \sigma$ level. The $K=0$ and $K=1$ transitions, however, only have a frequency difference of $4.25 \mathrm{MHz}\left(\sim 5.8 \mathrm{~km} \mathrm{~s}^{-1}\right)$. We used double-component Gaussian models to fit the $K=$ 0, 1 line profiles for $\mathrm{SW}$ and $\mathrm{NE}$, respectively, which give uncertainties of $10 \%$ for SW and $15 \%$ for NE in line fluxes. Another issue is that the velocity separation between $K=0$ and 1 transitions is comparable to the velocity difference of the emission peaks toward SW and NE. With our subarcsecond resolution, IRS $1 \mathrm{NE}$ and SW are only marginally separated, therefore the blending of the main $K=1$ emission from IRS 1 


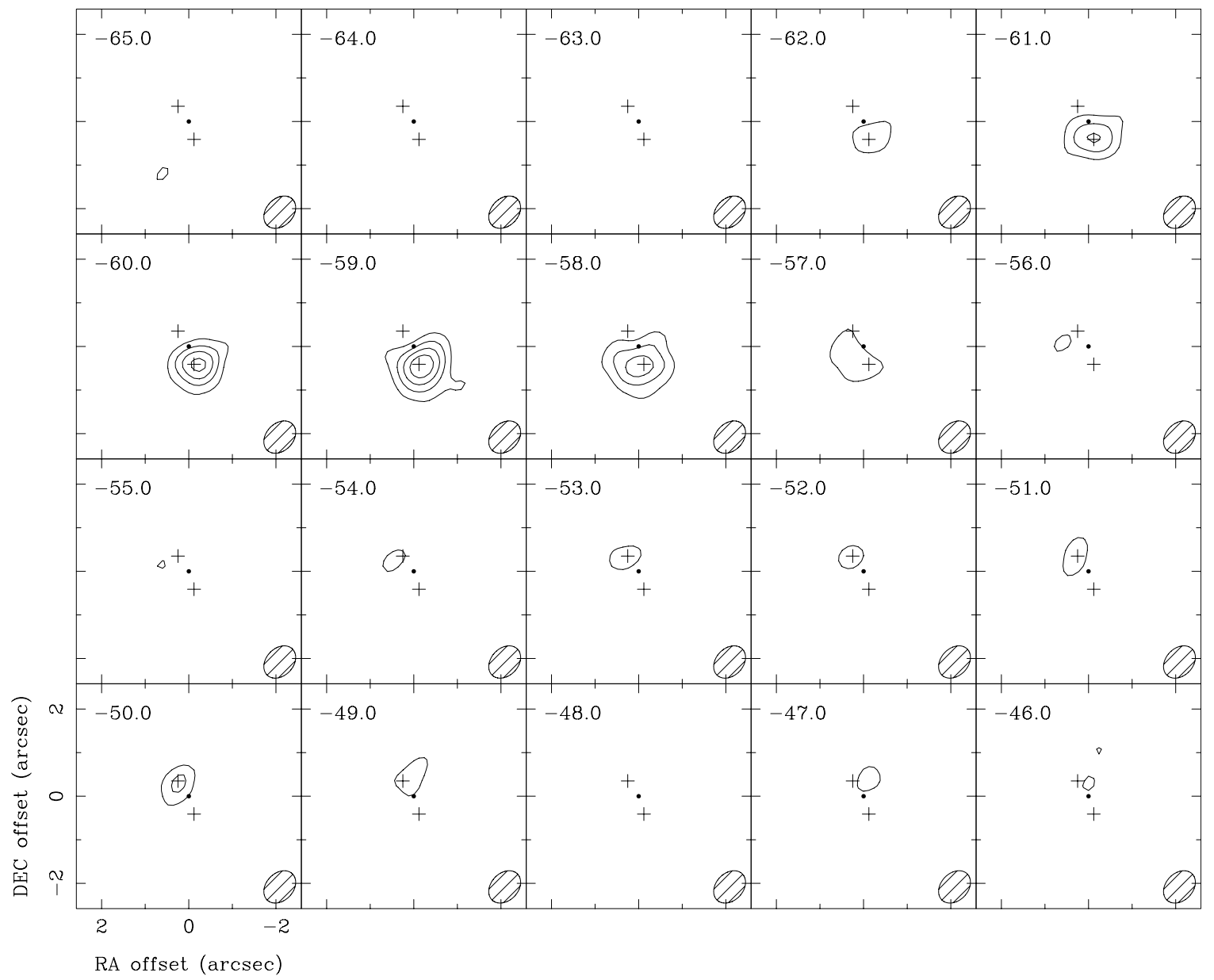

Figure 5. Channel maps of the OCS (19-18) lines. The contours are $(-1,1,2,3,4, \ldots) \times 5 \sigma$, and $1 \sigma=0.06 \mathrm{Jy}_{\text {beam }}^{-1}$. The FWHM beam size is $0^{\prime \prime} 8 \times 00^{\prime \prime} 6$, P.A. $=-43^{\circ}$. IRS 1 is located at the phase center (marked by filled black circles), and the intensity peaks of the SW and NE components are marked by plus signs.

SW with the redshifted $K=0$ emission from IRS $1 \mathrm{NE}$ is expected. Due to the contamination caused by the blending, the transition of $K=0$ toward IRS $1 \mathrm{NE}$ should be used with caution in the following analysis. As Figure 7(b) shows, the $K=0$ and $K=2$ transitions from IRS 1 SW are quite weak toward IRS 1 NE. Assuming that the main $K=1$ emission from IRS $1 \mathrm{SW}$ has a similar intensity, we estimate that about $15 \%$ of the observed intensity of the redshifted $K=0$ emission toward IRS $1 \mathrm{NE}$ is actually contributed by the blending from the main $K=1$ emission. Toward IRS $1 \mathrm{SW}$ the error of the main $K=1$ emission due to the blending from the redshifted $K=0$ emission is negligible.

The intensity of the $K=3$ component for both IRS 1 NE and $\mathrm{SW}$ is abnormally low compared to the other $K$ components under the assumption that the $\mathrm{CH}_{3} \mathrm{CN}$ components are in local thermal equilibrium (LTE), homogeneous, and optically thin. As shown in Figures 7(a) and (b) for the SW component, the peak intensity of the $K=0-4$ transitions are nearly identical, indicating that the low $K$ transitions of $\mathrm{CH}_{3} \mathrm{CN}$ in the SW component are optically thick. The optical depth of $K=3$ component is even higher due to its doubled statistical weight. Thus, the optically thick components must be excluded in the fitting for kinetic temperature with the assumption of optically thin and LTE (see Section 4.5).

In addition, we noticed that the intensity of the $K=6$ component toward IRS $1 \mathrm{NE}$ is higher than the other components including $K=3,4,5$, and 7 . This could be attributed to two causes. First, the $K=6$ component has a much higher column density than the components of $K=4$ and 5 due to its doubled statistic weight, but it still remains optically thin compared to the components of $K=0,1,2$, and 3 . Second, the intensity of the $K=6$ component toward IRS $1 \mathrm{NE}$ is likely to be affected by the line blending with $\operatorname{HNCO}\left(10_{1,9}-9_{1,8}\right)$ at rest frequency $v_{0}=220.5848 \mathrm{GHz}$ from $\mathrm{SW}$ and $\mathrm{CH}_{3}^{13} \mathrm{CN}\left(12_{3}-11_{3}\right)$ at $v_{0}=220.6000 \mathrm{GHz}$ from NE itself. From the comparison of Figures 7(b) and (d), it seems that the possible line blending caused a non-zero baseline in the frequency range from 220.58 to $220.61 \mathrm{GHz}$ toward NE, which might contribute up to $20 \%$ to the measured peak intensity of the $K=6$ component of $\mathrm{CH}_{3} \mathrm{CN}(12-11)$.

The possible blending from weaker molecular lines is also examined. We noticed that the line frequencies of $\mathrm{C}_{2} \mathrm{H}_{5} \mathrm{OH}$ are close to the $K=1,3$, and 4 transitions of $\mathrm{CH}_{3} \mathrm{CN}(12-11)$ and a $\mathrm{CH}_{3} \mathrm{OCHO}-\mathrm{E}$ line close to $K=3$ transition of $\mathrm{CH}_{3} \mathrm{CN}(12-11)$. However, according to the JPL catalog, the typical intensities of those lines are only a few percent of the $\mathrm{CH}_{3} \mathrm{CN}$ lines, and the contamination would produce no significant effects.

We constructed line images for each $K$ transition (not shown in this paper) and fitted the intensity peak positions for both the IRS $1 \mathrm{SW}$ and NE components based on the integrated line flux images of $K=0-8$. We also fitted the line profiles of each $K$ transition to determine their peak intensities $\left(\Delta S_{\mathrm{L}}\right)$, centroid velocity $\left(V_{\mathrm{LSR}}\right)$, and FWHM line width $\left(\Delta V_{\mathrm{FWHM}}\right)$. The derived values for these quantities are summarized in Table 4.

Taking the strong transitions $K=2-5$, with no blending, we combined the visibility data of the four transitions in 

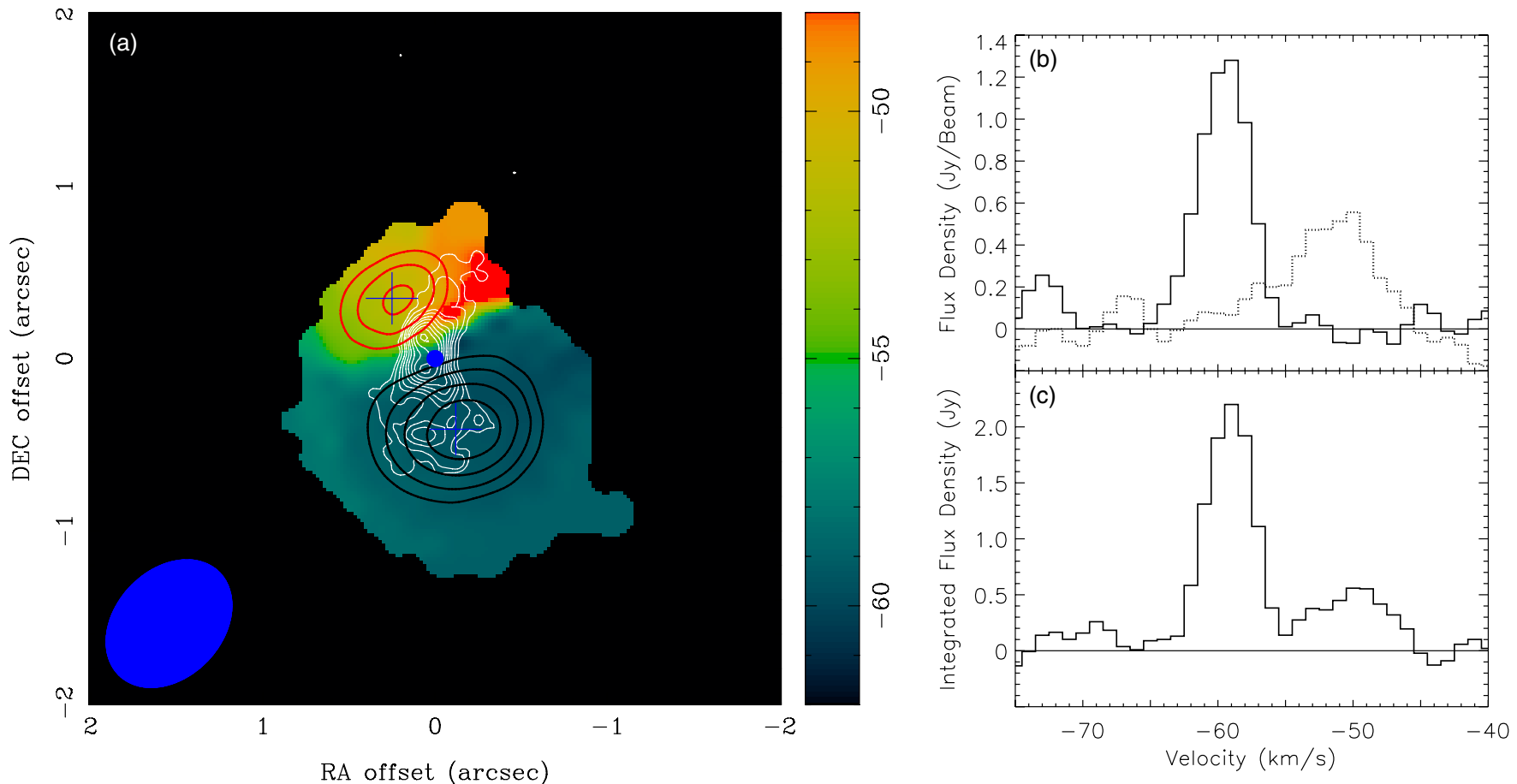

Figure 6. (a) The integrated intensity maps of the OCS(19-18) components IRS 1 NE (red) and SW (black) superimposed on the centroid velocity image (color background). The velocity ranges of the integrations are $(-54.0,-47.0) \mathrm{km} \mathrm{s}^{-1}$ and $(-62.5,-57.0) \mathrm{km} \mathrm{s}^{-1}$ for IRS $1 \mathrm{NE}$ and SW, respectively. The contour levels are $(2.3,2.8,3.3)$ and $(3.0,3.8,4.6,5.4) \mathrm{Jy} \mathrm{beam}^{-1} \mathrm{~km} \mathrm{~s}^{-1}$ for IRS $1 \mathrm{NE}$ and SW, respectively. The rms noise $\sigma$ is $0.15 \mathrm{Jy} \mathrm{beam}^{-1} \mathrm{~km} \mathrm{~s}^{-1}$. The FWHM beam size is $0^{\prime \prime} 8 \times$ 0.6 , P.A. $=-45^{\circ}$. The unit of the wedge scaling is $\mathrm{km} \mathrm{s}^{-1}$. The white contours represent the $1.3 \mathrm{~cm}$ continuum emission from the VLA observations (reproduced according to Gaume et al. 1995). IRS 1 is located at the phase center, marked by blue dot. Plus signs mark the peak positions of IRS 1 NE and SW in OCS(19-18). (b) The line profiles of OCS(19-18) at the peak positions of IRS 1 SW (solid line) and 1 NE (dashed line). (c) The line profile of OCS(19-18) integrated over the entire IRS 1 region.

(A color version of this figure is available in the online journal.)

Table 4

Properties of Hot Molecular Components in IRS 1

\begin{tabular}{|c|c|c|c|c|c|c|c|c|c|c|}
\hline Transition & $\begin{array}{c}v \\
(\mathrm{GHz})\end{array}$ & $\begin{array}{c}\Delta \alpha, \Delta \delta \\
\quad\left({ }^{\prime \prime}\right)\end{array}$ & $\begin{array}{c}\Delta S_{\mathrm{L}} \\
\left(\mathrm{Jy} \mathrm{beam}^{-1}\right)\end{array}$ & $\begin{array}{c}V_{\mathrm{LSR}} \\
\left(\mathrm{km} \mathrm{s}^{-1}\right)\end{array}$ & $\begin{array}{l}\Delta V_{\mathrm{FWHM}} \\
\left(\mathrm{km} \mathrm{s}^{-1}\right)\end{array}$ & $\begin{array}{l}T_{\mathrm{B}} d v(\text { peak }) \\
\left(\mathrm{K} \mathrm{km} \mathrm{s}^{-1}\right)\end{array}$ & $\begin{array}{l}N_{J K}^{\mathrm{obs}} \\
\quad\left(10^{13}\right.\end{array}$ & $\begin{array}{c}N_{J K}^{\text {corr }} \\
\left.\mathrm{cm}^{-2}\right)\end{array}$ & $g_{J K}$ & $\begin{array}{c}E_{J K} / k \\
(\mathrm{~K})\end{array}$ \\
\hline \multicolumn{11}{|c|}{ IRS $1 \mathrm{SW}$} \\
\hline \multicolumn{11}{|l|}{$\mathrm{CH}_{3} \mathrm{CN}$} \\
\hline $12_{0}-11_{0}$ & 220.747 & $-0.16 \pm 0.04,-0.44 \pm 0.03$ & $1.4 \pm 0.1$ & $-59.3 \pm 0.1$ & $4.4 \pm 0.2$ & $282 \pm 22$ & $2.9 \pm 0.2$ & $\ldots$ & 100 & 68 \\
\hline $12_{1}-11_{1}$ & 220.743 & $-0.15 \pm 0.06,-0.45 \pm 0.05$ & $1.4 \pm 0.1$ & $-59.2 \pm 0.1$ & $3.9 \pm 0.3$ & $258 \pm 21$ & $2.7 \pm 0.2$ & & 100 & 76 \\
\hline $12_{2}-11_{2}$ & 220.730 & $-0.11 \pm 0.04,-0.45 \pm 0.03$ & $1.4 \pm 0.1$ & $-59.6 \pm 0.1$ & $4.2 \pm 0.2$ & $260 \pm 24$ & $2.8 \pm 0.3$ & $\ldots$ & 100 & 97 \\
\hline $12_{3}-11_{3}$ & 220.709 & $-0.21 \pm 0.04,-0.48 \pm 0.03$ & $1.4 \pm 0.1$ & $-59.6 \pm 0.1$ & $4.3 \pm 0.1$ & $282 \pm 18$ & $2.8 \pm 0.2$ & $\ldots$ & 200 & 133 \\
\hline $12_{4}-11_{4}$ & 220.679 & $-0.07 \pm 0.03,-0.43 \pm 0.03$ & $1.2 \pm 0.1$ & $-59.5 \pm 0.1$ & $4.2 \pm 0.2$ & $249 \pm 23$ & $2.8 \pm 0.3$ & $3.3 \pm 0.3$ & 100 & 183 \\
\hline $12_{5}-11_{5}$ & 220.641 & $-0.04 \pm 0.04,-0.41 \pm 0.03$ & $0.9 \pm 0.1$ & $-59.5 \pm 0.1$ & $4.6 \pm 0.3$ & $201 \pm 26$ & $2.5 \pm 0.3$ & $2.8 \pm 0.4$ & 100 & 247 \\
\hline $12_{6}-11_{6}$ & 220.594 & $-0.01 \pm 0.04,-0.30 \pm 0.03$ & $0.9 \pm 0.1$ & $-59.5 \pm 0.1$ & $4.5 \pm 0.2$ & $205 \pm 20$ & $2.8 \pm 0.3$ & $3.2 \pm 0.3$ & 200 & 326 \\
\hline $12_{7}-11_{7}$ & 220.539 & $+0.08 \pm 0.09,-0.25 \pm 0.08$ & $0.5 \pm 0.1$ & $-59.5 \pm 0.2$ & $4.1 \pm 0.4$ & $97 \pm 23$ & $1.5 \pm 0.4$ & $1.5 \pm 0.4$ & 100 & 419 \\
\hline $12_{8}-11_{8}$ & 220.476 & $+0.06 \pm 0.10,-0.45 \pm 0.12$ & $0.3 \pm 0.1$ & $-59.3 \pm 0.2$ & $3.4 \pm 0.4$ & $54 \pm 12$ & $1.0 \pm 0.2$ & $1.0 \pm 0.2$ & 100 & 526 \\
\hline$(K=2-5)$ & & $-0.11 \pm 0.03,-0.44 \pm 0.02$ & $1.2 \pm 0.1$ & $-59.5 \pm 0.1$ & $4.3 \pm 0.1$ & $250 \pm 16$ & & $\ldots$ & & $\ldots$ \\
\hline \multicolumn{11}{|c|}{ 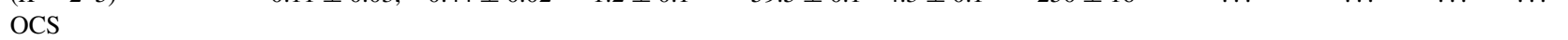 } \\
\hline $19-18$ & 231.061 & $-0.13 \pm 0.03,-0.41 \pm 0.03$ & $1.3 \pm 0.1$ & $-59.5 \pm 0.1$ & $4.4 \pm 0.1$ & $267 \pm 15$ & $\ldots$ & $\ldots$ & $\ldots$ & $\ldots$ \\
\hline \multicolumn{11}{|c|}{ IRS $1 \mathrm{NE}$} \\
\hline \multicolumn{11}{|l|}{$\mathrm{CH}_{3} \mathrm{CN}$} \\
\hline $12_{0}-11_{0}$ & 220.747 & $+0.39 \pm 0.15,+0.26 \pm 0.25$ & $0.6 \pm 0.1$ & $-52.0 \pm 0.2$ & $5.4 \pm 0.5$ & $164 \pm 22$ & $1.4 \pm 0.2$ & $1.5 \pm 0.2$ & 100 & 69 \\
\hline $12_{1}-11_{1}$ & 220.743 & $+0.21 \pm 0.03,+0.33 \pm 0.03$ & $0.5 \pm 0.1$ & $-51.4 \pm 0.4$ & $5.4 \pm 0.5$ & $138 \pm 24$ & $1.4 \pm 0.2$ & $1.5 \pm 0.3$ & 100 & 76 \\
\hline $12_{2}-11_{2}$ & 220.730 & $+0.23 \pm 0.04,+0.36 \pm 0.06$ & $0.4 \pm 0.1$ & $-51.4 \pm 0.3$ & $6.0 \pm 0.7$ & $109 \pm 23$ & $1.2 \pm 0.2$ & $1.2 \pm 0.3$ & 100 & 97 \\
\hline $12_{3}-11_{3}$ & 220.709 & $+0.26 \pm 0.06,+0.28 \pm 0.07$ & $0.3 \pm 0.1$ & $-51.0 \pm 0.3$ & $5.7 \pm 0.6$ & $96 \pm 14$ & $1.1 \pm 0.2$ & $\ldots$ & 200 & 133 \\
\hline $12_{4}-11_{4}$ & 220.679 & $+0.14 \pm 0.15,+0.35 \pm 0.16$ & $0.3 \pm 0.1$ & $-51.3 \pm 0.3$ & $4.4 \pm 0.7$ & $61 \pm 18$ & $0.7 \pm 0.2$ & $0.7 \pm 0.2$ & 100 & 183 \\
\hline $12_{5}-11_{5}$ & 220.641 & $+0.31 \pm 0.06,+0.12 \pm 0.08$ & $0.3 \pm 0.1$ & $-52.0 \pm 0.5$ & $4.1 \pm 0.6$ & $49 \pm 15$ & $0.6 \pm 0.2$ & $0.6 \pm 0.2$ & 100 & 247 \\
\hline $12_{6}-11_{6}$ & 220.594 & $+0.24 \pm 0.06,+0.27 \pm 0.08$ & $0.4 \pm 0.1$ & $-50.8 \pm 0.2$ & $3.4 \pm 0.2$ & $60 \pm 10$ & $0.8 \pm 0.1$ & $0.9 \pm 0.2$ & 200 & 326 \\
\hline $12_{7}-11_{7}$ & 220.539 & $+0.42 \pm 0.15,+0.06 \pm 0.16$ & $0.2 \pm 0.1$ & $-51.1 \pm 0.3$ & $2.8 \pm 0.5$ & $26 \pm 11$ & $0.4 \pm 0.2$ & $0.4 \pm 0.2$ & 100 & 419 \\
\hline$(K=2-5)$ & & $+0.23 \pm 0.04,+0.33 \pm 0.04$ & $0.3 \pm 0.1$ & $-51.4 \pm 0.2$ & $6.8 \pm 0.5$ & $103 \pm 14$ & 然 & (6) & & \\
\hline \multicolumn{11}{|l|}{ OCS } \\
\hline 19-18 & 231.061 & $+0.25 \pm 0.03,+0.35 \pm 0.02$ & $0.5 \pm 0.1$ & $-51.5 \pm 0.3$ & $7.2 \pm 0.6$ & $180 \pm 30$ & $\ldots$ & $\ldots$ & $\cdots$ & $\ldots$ \\
\hline
\end{tabular}

Note. The reference position is R.A. $(\mathrm{J} 2000)=23^{\mathrm{h}} 13^{\mathrm{m}} 45^{\mathrm{s}} \cdot 37$, decl. $(\mathrm{J} 2000)=61^{\circ} 28^{\prime} 10^{\prime \prime} 43$. 

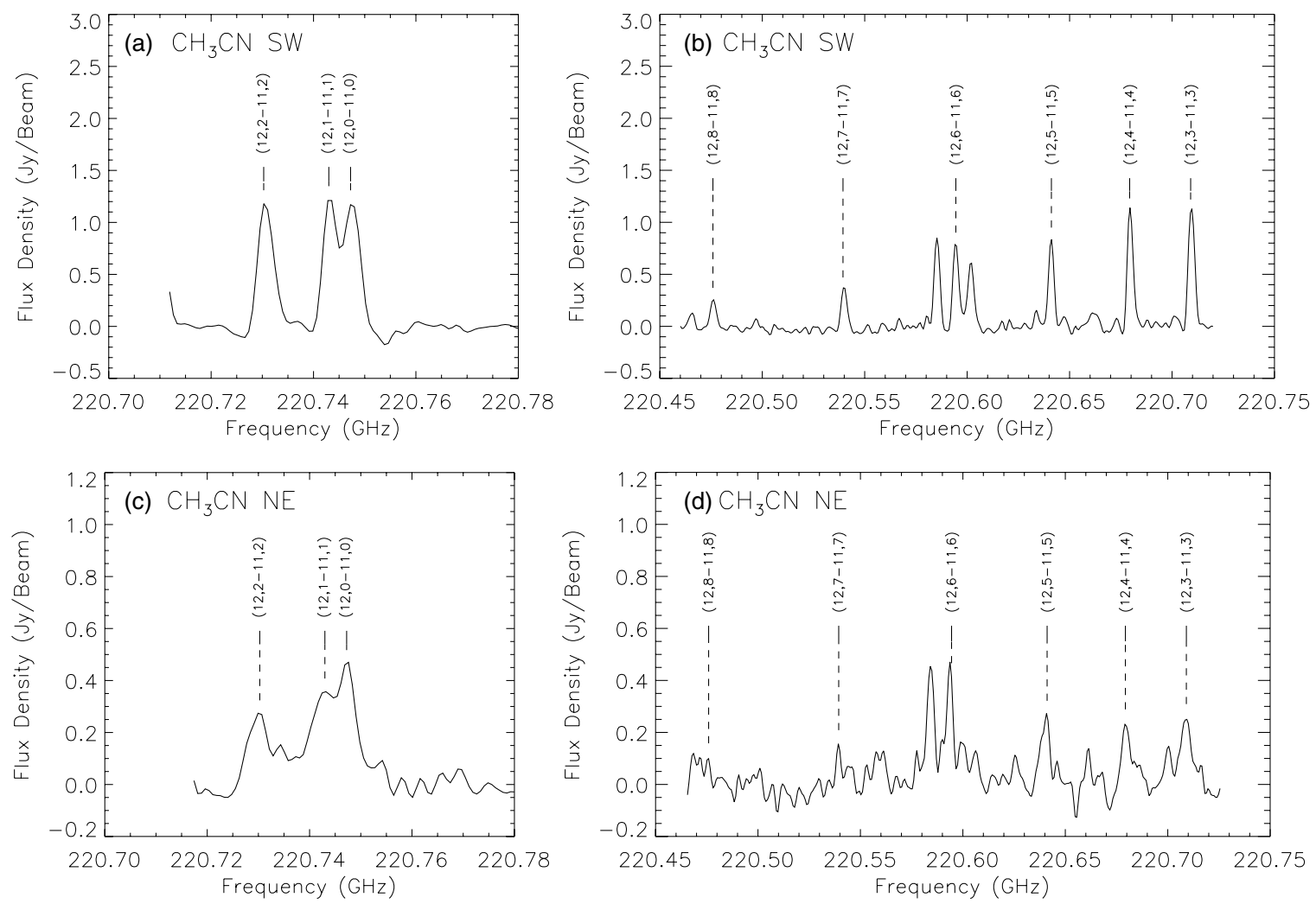

Figure 7. (a)-(b) The line profiles of $\mathrm{CH}_{3} \mathrm{CN}(12-11), K=0,1,2$, and $K=3,4,5,6,7,8$ at the intensity peak position of IRS $1 \mathrm{SW}$. The spectra are converted to $V_{\mathrm{LSR}}=-59.5 \mathrm{~km} \mathrm{~s}^{-1}$. The blending due to component NE is neglectable at this position. (c)-(d) The line profiles of $\mathrm{CH}_{3} \mathrm{CN}(12-11), K=0,1,2$, and $K=3,4,5,6,7,8$ at the intensity peak position of IRS 1 NE. The spectra are converted to $V_{\mathrm{LSR}}=-51.5 \mathrm{~km} \mathrm{~s}^{-1}$. In (c) the transition $K=0$ is blended with the transition $K=1$ from IRS 1 SW.

the LSR-velocity domain to construct the channel maps of $\mathrm{CH}_{3} \mathrm{CN}(12-11)$ (Figure 8), which is equivalent to averaging the line intensities from the $K=2-5$ transitions. This spectral line image is hereafter referred to as the $K=2-5$ line image. Figure 9(a) shows the integrated line intensity image of IRS 1 SW and NE made from the $K=2-5$ line image, which is in good agreement with that of OCS(19-18) (see Figure 6(a)). The peak position of IRS $1 \mathrm{SW}$ is $\left(-0^{\prime} \cdot 11 \pm 00^{\prime} 03,-0{ }^{\prime} 44 \pm 00^{\prime} 03\right)$ offset from IRS 1, based on a Gaussian fitting, while IRS 1 NE is offset by (0'.23 $\left.\pm 00^{\prime} 07,0{ }^{\prime} 33 \pm 00^{\prime} \cdot 09\right)$, consistent with OCS(19-18). The centroid velocity distribution made from the $\mathrm{CH}_{3} \mathrm{CN}(12-11)$ $K=2-5$ line image is also shown in Figure 9(a), showing a similar velocity gradient from $\mathrm{SW}$ to $\mathrm{NE}$ as that derived from the OCS(19-18) line (see Figure 6(a)).

Figure 9(b) shows the spectral profiles made from the $K=$ 2-5 line image of $\mathrm{CH}_{3} \mathrm{CN}(12-11)$ toward the peak positions of IRS 1 SW and NE, while Figure $9(\mathrm{c})$ is the spectrum integrated over the entire IRS 1 region. Gaussian fitting gives $V_{\mathrm{LSR}}=-59.5 \pm 0.1 \mathrm{~km} \mathrm{~s}^{-1}$ and $\Delta V_{\mathrm{FWHM}}=4.3 \pm 0.1 \mathrm{~km} \mathrm{~s}^{-1}$ for IRS 1 SW, and $V_{\mathrm{LSR}}=-51.4 \pm 0.2 \mathrm{~km} \mathrm{~s}^{-1}$ and $\Delta V_{\mathrm{FWHM}}=$ $6.8 \pm 0.5 \mathrm{~km} \mathrm{~s}^{-1}$ for IRS $1 \mathrm{NE}$, respectively. These results are in good agreement with those from OCS(19-18).

In addition, a weak (more than $3 \sigma, 1 \sigma=0.03 \mathrm{Jy}^{\text {beam }}{ }^{-1}$ ) absorption of the $\mathrm{CH}_{3} \mathrm{CN}(12-11)$ line at $V_{\mathrm{LSR}}=-56 \mathrm{~km} \mathrm{~s}^{-1}$ was observed from the $K=2-5$ line image (see Figures 8 and $9(\mathrm{c}))$. We will further discuss these possible absorption features.

\subsection{Kinetic Temperature of the Hot Molecular Gas}

As a symmetric top molecule, $\mathrm{CH}_{3} \mathrm{CN}$ is a good probe for the determination of the kinetic temperature of the molecular gas. The multiple $K$ transitions for $J \rightarrow J-1$ in a rotational transition of $\mathrm{CH}_{3} \mathrm{CN}$ can be observed simultaneously, and their line flux ratios reflect the rotational temperature $T_{\text {rot }}$, equivalent to the kinetic temperature $T_{\mathrm{k}}$ under LTE condition. Using rotational temperature equilibrium analysis (e.g., Hollis 1982; Loren \& Mundy 1984; Churchwell et al. 1992; Goldsmith \& Langer 1999; Araya et al. 2005; Furuya et al. 2008), one can determine the kinetic (rotation) temperature $T_{\text {rot }}$ and total column density $N$ of the hot molecular gas in an optically thin case by fitting a linear function to the relation between the (logarithmic) column density $N_{J K}$ and the rotational energy $E_{J K}$ at levels $(J, K)$ of $\mathrm{CH}_{3} \mathrm{CN}$ :

$$
\ln \left(\frac{N_{J K}}{g_{J K}}\right)=\ln \left[\frac{N}{Q\left(T_{\mathrm{rot}}\right)}\right]-\ln \left[\frac{\tau_{J, K}}{1-e^{-\tau_{J, K}}}\right]-\frac{E_{J K}}{k T_{\mathrm{rot}}},
$$

where $g_{J K}$ is the statistical weight of the level $(J, K), N$ is the total column density, $Q\left(T_{\text {rot }}\right)=3.89 T_{\text {rot }}^{1.5} /\left(1-\exp \left(-524.8 / T_{\text {rot }}\right)\right)^{2}$ is the partition function, and $k$ is Boltzmann constant.

In the case of NGC 7538 IRS 1 , some of the $K$ components of $\mathrm{CH}_{3} \mathrm{CN}(12-11)$ are obviously optically thick (see Section 4.4). Therefore, we made iterations in fitting the rotation temperature. First, higher $K$ components ( $K=4,5,7$, and 8 for SW, and $K=$ 4,5 , and 7 for NE) were assumed to be optically thin and used in the initial fitting with Equation (1) by eliminating the opticaldepth term $-\ln \left(\tau_{J, K} /\left(1-\exp \left(-\tau_{J, K}\right)\right)\right)$. The initial fitting gave a rotation temperature $T_{\text {rot }}^{\prime}$ and a total column density $N^{\prime}$, with which the corresponding optical depths $\tau_{J, K}^{\prime}$ and optical-depthcorrection factors $f_{\tau}^{\prime}(J, K)=\tau_{J, K} /\left(1-\exp \left(-\tau_{J, K}\right)\right)$ were derived for each $K$ component. In the following iterations, we used $f_{\tau}^{\prime}$ to correct the observed surface column densities, $N_{J K}^{\mathrm{obs}}$, and added the other $K$ components to the fitting. To avoid the bias caused by the optically thick $K$ components, their weighting 


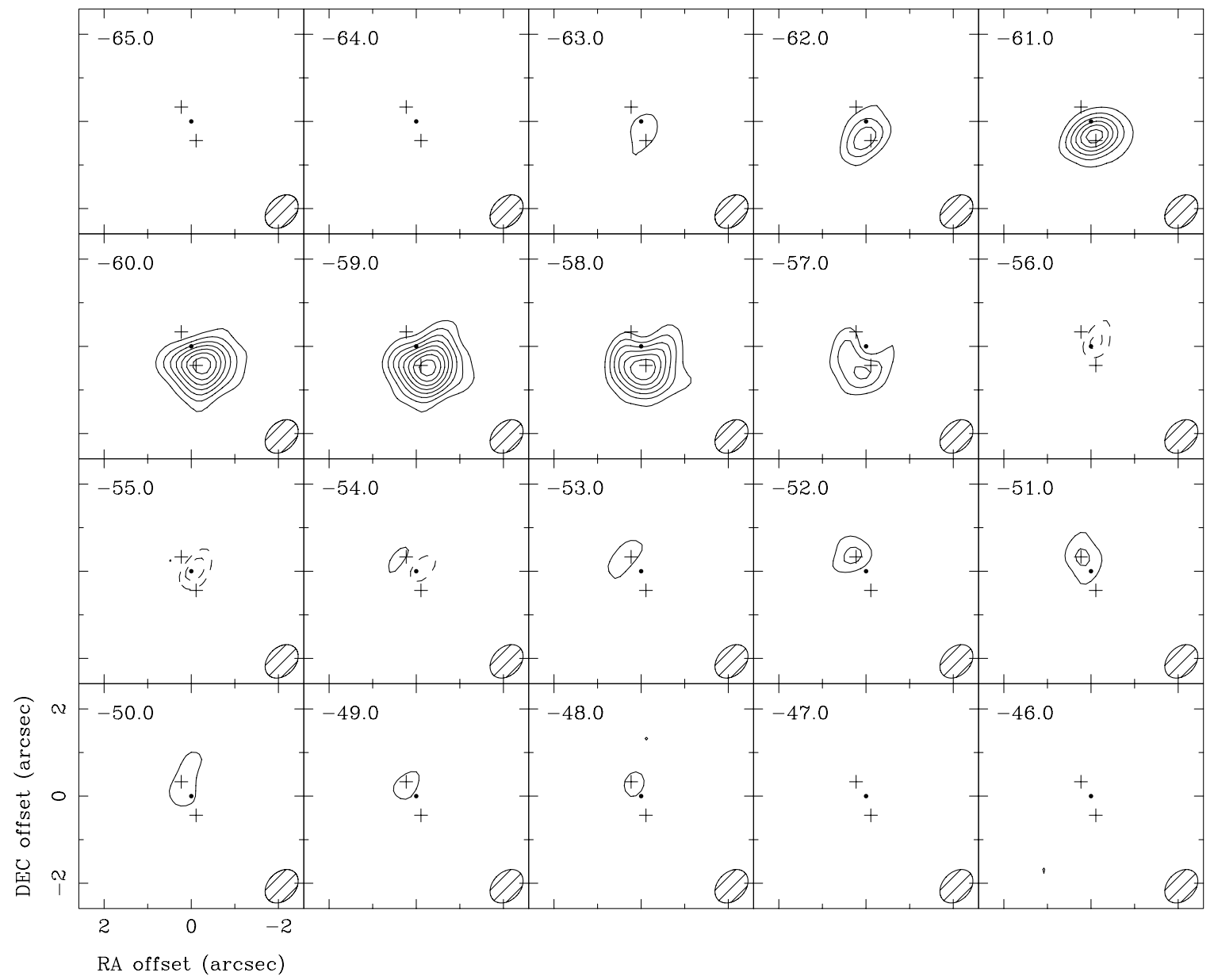

Figure 8. Channel maps of $\mathrm{CH}_{3} \mathrm{CN}(12-11)$ were constructed by combining the visibility data of $K=2-5$ transitions in the LSR-velocity domain, which is equivalent to averaging the line intensities from the $K=2-5$ transitions. The contours are $(-1,1,2,3,4, \ldots) \times 5 \sigma$, and $1 \sigma=0.03 \mathrm{Jy} \mathrm{beam}^{-1}$. The FWHM beam size is $0{ }^{\prime \prime} 8 \times$ $0 . ' 6$, P.A. $=-45^{\circ}$. IRS 1 is located at the phase center (indicated by filled black circles), and the intensity peaks of the SW and NE components are marked by plus signs.

in the fitting was inversely proportional to their deviations from the fitting curve of the previous iteration.

The final least-square fitting results are shown in Figure 10, giving rotational temperatures of $260 \pm 30 \mathrm{~K}$ and $260 \pm 60 \mathrm{~K}$ for IRS $1 \mathrm{SW}$ and NE, respectively. The derived rotational temperatures are consistent with $297 \mathrm{~K}$ reported by Klaassen et al. (2009) and $245 \mathrm{~K}$ reported by Qiu et al. (2011). We note that the optically thick components, $K=0,1,2$, and 3 for IRS $1 \mathrm{SW}$ and $K=3$ for NE, significantly deviate from the fitting curve, and have little influence on the fitting results due to their low weighting. The derived optical-depth-correction factors $f_{\tau}(J, K)$, which are based on the fitting results $(N$ and $T_{\text {rot }}$ ), are not adequate to correct the $N_{J K}$ to the fitting curve. The optical depths of the $K$ components used in the initial iterations of the fitting are non-negligible.

For the high $K$ components with smaller optical depths, the correction factor $f_{\tau}$ is close to unity and not sensitive to $\tau$, while for the optically thick components, $f_{\tau}$ is almost proportional to $\tau$. An underestimation of the $\tau_{J, K}$ of the high $K$ components will not change the slope of the fit, which is largely determined by these levels. However, the fitted total column density will cause significant underestimations for the $\tau_{J, K}$ and $f_{\tau}(J, K)$ of the optically thick levels, which results in the large deviation from the fit (see Figure 10).

In summary, by fitting the $K$ components of $\mathrm{CH}_{3} \mathrm{CN}(12-11)$ lines with small optical depths, we estimated the kinetic temperature of the hot molecular clumps to be $\sim 260 \mathrm{~K}$. The corre- sponding total column densities for IRS $1 \mathrm{SW}$ and NE, however, might be underestimated by a factor of a few and represent the lower limits. With the derived $T_{\text {rot }}$, we can estimate the lower limits of the total column densities $N=1.9 \pm 0.5 \times 10^{16} \mathrm{~cm}^{-2}$ and $N=3.5 \pm 1.5 \times 10^{15} \mathrm{~cm}^{-2}$ toward the intensity peaks of $\mathrm{SW}$ and NE, respectively. With an abundance of $1 \times 10^{-8}$ for $\mathrm{CH}_{3} \mathrm{CN}$ (Qiu et al. 2011, and references therein), these column densities correspond to gas masses of 4 and $0.7 M_{\odot}$ for SW and NE (in one FWHM beam size), respectively.

\section{6. ${ }^{13} \mathrm{CO}(2-1), \mathrm{CO}(2-1)$, and $\mathrm{HCN}(1-0)$ toward IRS 1 \\ 4.6.1. ${ }^{13} \mathrm{CO}(2-1)$ toward IRS 1}

The ${ }^{13} \mathrm{CO}(2-1)$ line is a good tracer for the gas with a low to intermediate density. We produced a high-velocity $\left(\delta V=0.5 \mathrm{~km} \mathrm{~s}^{-1}\right)$ and high-angular $\left(0{ }^{\prime} .8 \times 0^{\prime \prime} \cdot 6\right)$ resolution image of the ${ }^{13} \mathrm{CO}(2-1)$ line to study both the absorption line against the continuum source and the emission line from the molecular gas immediately surrounding IRS 1 . Figure 11 shows the channel maps of ${ }^{13} \mathrm{CO}(2-1)$, exhibiting a strong absorption between -60 and $-53 \mathrm{~km} \mathrm{~s}^{-1}$ toward the $1.3 \mathrm{~mm}$ continuum peak and an emission component SW to IRS 1 in the velocity range of $(-63,-60) \mathrm{km} \mathrm{s}^{-1}$. A similar distribution of ${ }^{13} \mathrm{CO}(2-1)$ gas is also shown in Figure 12, which features the integrated intensity maps of both ${ }^{13} \mathrm{CO}(2-1)$ emission and absorption from the velocity range between -43 to $-66 \mathrm{~km} \mathrm{~s}^{-1}$. 

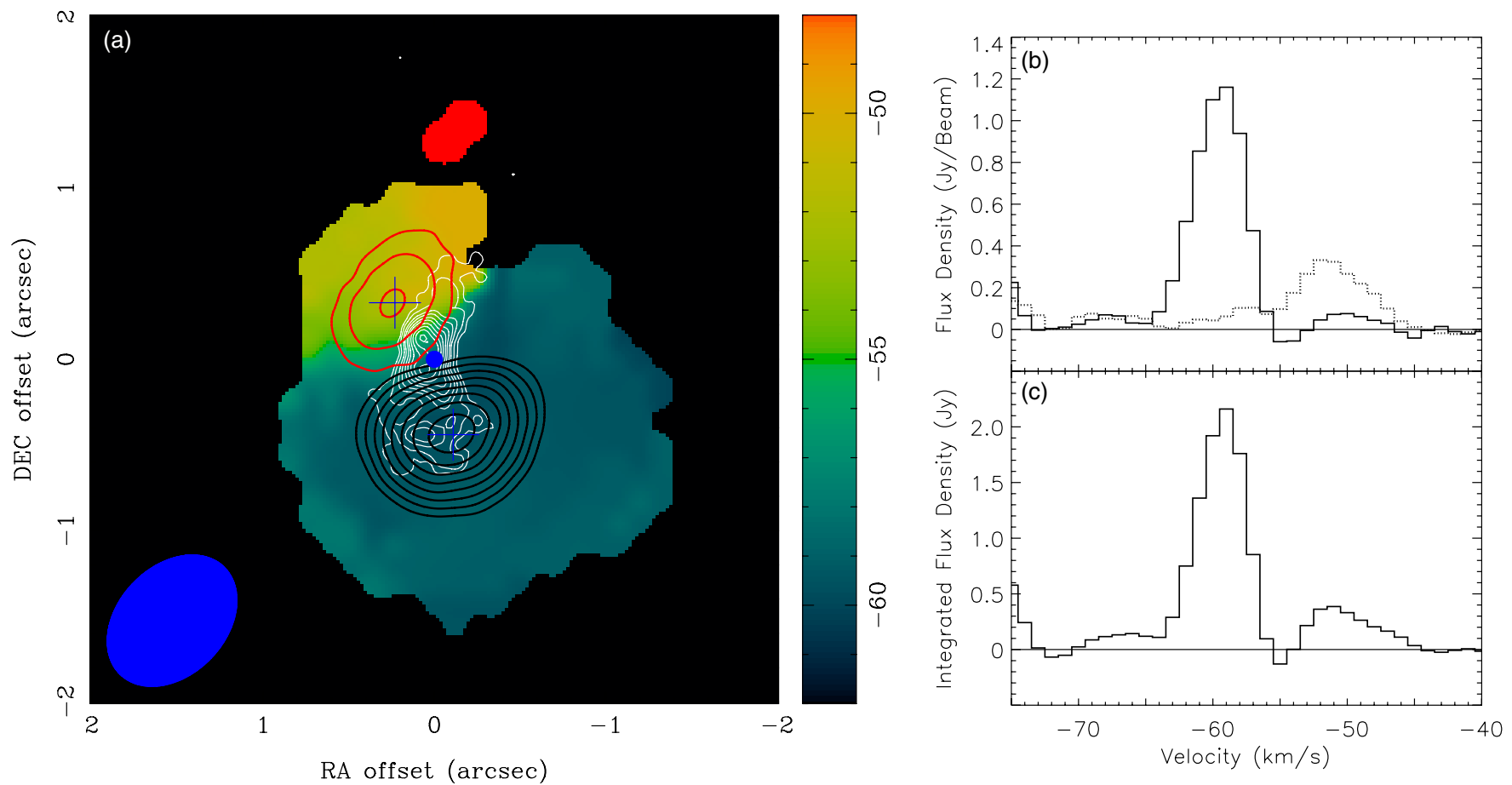

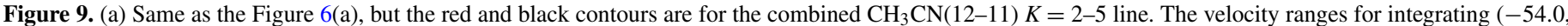

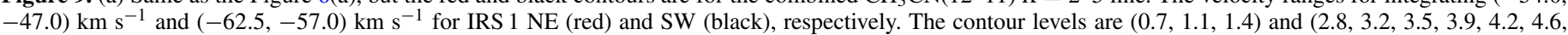

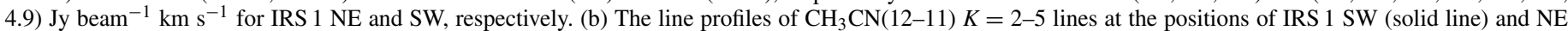
(dashed line). (c) The line profile of $\mathrm{CH}_{3} \mathrm{CN}(12-11) K=2-5$ lines integrated over the entire IRS 1 region.

(A color version of this figure is available in the online journal.)

The morphology of the integrated ${ }^{13} \mathrm{CO}(2-1)$ emission shows an elongated feature across IRS 1 with a P.A. $\sim 35^{\circ}$. At an offset $\sim 1^{\prime \prime}$ southwest of IRS 1 , a blueshifted gas component is also observed from ${ }^{13} \mathrm{CO}(2-1)$ emission. In general, both the integrated line flux and the intensity-weighted velocity images derived from the ${ }^{13} \mathrm{CO}(2-1)$ line appear to be in good agreement with the results observed from the molecular lines OCS(19-18) and $\mathrm{CH}_{3} \mathrm{CN}(12-11)$.

We made a ${ }^{13} \mathrm{CO}(2-1)$ spectrum toward the $1.3 \mathrm{~mm}$ emission peak in IRS 1 region and fitted the line profiles with a multiple-Gaussian-component model (see Figure 13(a)). A narrow $\left(\Delta V_{\mathrm{FWHM}} \sim 1 \mathrm{~km} \mathrm{~s}^{-1}\right)$ and strong $\left(\Delta S_{\mathrm{L}}=-2.6 \mathrm{Jy}\right.$ beam $\left.^{-1}\right)$ absorption feature at $-59.0 \mathrm{~km} \mathrm{~s}^{-1}$ was observed toward IRS 1, which is best seen in a spectrum made from using the long $(>100 \mathrm{k} \lambda)$ baseline data. The narrow velocity characteristics of this feature suggest that a large fraction of the absorption at the velocity close to the systemic velocity arises from the absorption by the cold gas in the molecular envelope located in front of the continuum source IRS 1 . The absorption peak velocity in ${ }^{13} \mathrm{CO}(2-1)$ verifies that the systemic velocity of IRS 1 is very close (within $0.5 \mathrm{~km} \mathrm{~s}^{-1}$ ) to $V_{\text {sys }}=-59.0 \mathrm{~km} \mathrm{~s}^{-1}$ as observed in other molecular lines. Given $\Delta S_{\mathrm{L}}=-2.6 \mathrm{Jy}$ beam $^{-1}$ and $S_{\mathrm{C}}=3.25 \mathrm{Jy}_{\text {beam }}{ }^{-1}$ the peak optical depth of the ${ }^{13} \mathrm{CO}(2-1)$ line can be determined for the main absorption feature near the systemic velocity using Equation (1) of Qin et al. (2008), $\tau_{\mathrm{L}}=-\ln \left(1+\Delta S_{\mathrm{L}} / S_{\mathrm{C}}\right)=1.6$.

Two more absorption components are seen at -57.0 and $-54.0 \mathrm{~km} \mathrm{~s}^{-1}$, which are likely due to infall toward the central source in IRS 1. For these two absorption features, the equivalent total line width is $\sim 5 \mathrm{~km} \mathrm{~s}^{-1}$, which is consistent with the main absorption feature in the inverse P-Cygni profile suggested from the lower-angular resolution observations of the $\mathrm{HCO}^{+}(1-0)$ line (Corder 2008; Sandell et al. 2009). A weak
( $\left.0.5 \mathrm{Jy}_{\text {beam }}{ }^{-1}\right)$, and broad $\left(\Delta V_{\mathrm{FWHM}}=5.5 \mathrm{~km} \mathrm{~s}^{-1}\right)$ emission at velocity $-63.0 \mathrm{~km} \mathrm{~s}^{-1}$ was also detected, corresponding to the blueshifted emission in the inverse P-Cygni profile from lower angular resolution observations.

Two additional significant spectral features in ${ }^{13} \mathrm{CO}(2-1)$ were also detected: a blueshifted absorption component $\left(\Delta S_{\mathrm{L}}=\right.$ $-0.5 \mathrm{Jy} \mathrm{beam}{ }^{-1}, \Delta V_{\mathrm{FWHM}}=1.3 \mathrm{~km} \mathrm{~s}^{-1}$ ) at $V_{\mathrm{LSR}}=$ $-63.8 \mathrm{~km} \mathrm{~s}^{-1}$ and a redshifted emission component $\left(\Delta S_{\mathrm{L}}=\right.$ $\left.0.45 \mathrm{Jy} \mathrm{beam}^{-1}, \Delta V_{\mathrm{FWHM}}=2.5 \mathrm{~km} \mathrm{~s}^{-1}\right)$ at $V_{\mathrm{LSR}}=$ $-48.0 \mathrm{~km} \mathrm{~s}^{-1}$. The blueshifted absorption component is probably due to the foreground outflow gas, for which we find $\tau_{\mathrm{L}}=-\ln \left(1+\Delta S_{\mathrm{L}} / S_{\mathrm{C}}\right) \approx 0.17$. While the redshifted emission at $V_{\mathrm{LSR}}=-48.0 \mathrm{~km} \mathrm{~s}^{-1}$ could also be due to outflow, it more likely traces a background cloud emission at the same radial velocity, as indicated by lower-resolution studies on the general gas distribution in the NGC 7538 region (G. Sandell 2013, private communications).

To estimate the column density of the observed ${ }^{13} \mathrm{CO}$ gas, a reasonable excitation temperature $T_{\mathrm{ex}}$ is needed. The absorption feature of ${ }^{13} \mathrm{CO}(2-1)$ is seen only in the central $1 . .5$ region in projection. If the ${ }^{13} \mathrm{CO}$ gas is all within the central $1 . .5$, and coexists with the hot molecular gas, then a high $T_{\text {ex }}$ of $\sim 200 \mathrm{~K}$ is appropriate. However, the lack of ${ }^{13} \mathrm{CO}$ absorption outside the 1..5 region might simply be due to the absence of an emission background. In this case, the ${ }^{13} \mathrm{CO}$ absorption arises from gas along the line of sight, and a lower excitation temperature should be considered. Sandell \& Sievers (2004) derived a $T_{\mathrm{d}}$ of $75 \mathrm{~K}$ from fitting the dust emission in an $\sim 11^{\prime \prime} .5 \times 10^{\prime}$. 6 region around IRS 1 , constrained by the submillimeter luminosity of IRS 1 . We calculated the total ${ }^{13} \mathrm{CO}$ column density with both the high $T_{\text {ex }}=200 \mathrm{~K}$ (upper limit) and low $T_{\mathrm{ex}}=75 \mathrm{~K}$ (lower limit) for each velocity component of ${ }^{13} \mathrm{CO}(2-1)$ and listed the results in Table 5. 
Table 5

${ }^{13} \mathrm{CO}(2-1), \mathrm{CO}(2-1)$, and $\mathrm{HCN}(1-0)$ toward IRS 1

\begin{tabular}{|c|c|c|c|c|c|c|c|}
\hline Source & $\begin{array}{c}\Delta \alpha, \Delta \delta \\
\text { “,”, }\end{array}$ & $\begin{array}{l}\Delta S_{\mathrm{L}} \\
(\mathrm{Jy})\end{array}$ & $\begin{array}{c}V_{\mathrm{LSR}} \\
\left(\mathrm{km} \mathrm{s}^{-1}\right)\end{array}$ & $\begin{array}{l}\Delta V_{\text {FWHM }} \\
\left(\mathrm{km} \mathrm{s}^{-1}\right)\end{array}$ & $\tau_{\mathrm{L}}$ & $\begin{array}{l}N_{13}{ }_{13}(200)^{\mathrm{a}} \\
\left(10^{15} \mathrm{~cm}^{-2}\right)\end{array}$ & $\begin{array}{l}N_{13} \mathrm{CO}(75)^{\mathrm{b}} \\
\left(10^{15} \mathrm{~cm}^{-2}\right)\end{array}$ \\
\hline \multicolumn{8}{|c|}{${ }^{13} \mathrm{CO}(2-1)$ with a beam area $0^{\prime \prime} 8 \times 0^{\prime \prime} .6$} \\
\hline IRS 1 & $+0.0,+0.0$ & $\begin{array}{r}-0.5 \pm 0.1 \\
0.5 \pm 0.1 \\
-2.6 \pm 0.1 \\
-2.3 \pm 0.1 \\
-2.0 \pm 0.1 \\
0.5 \pm 0.1\end{array}$ & $\begin{array}{l}-63.8 \pm 0.3 \\
-63.0 \pm 0.3 \\
-59.0 \pm 0.3 \\
-57.0 \pm 0.3 \\
-54.5 \pm 0.3 \\
-48.0 \pm 0.3\end{array}$ & $\begin{array}{l}1.3 \pm 0.2 \\
5.5 \pm 0.4 \\
1.5 \pm 0.2 \\
2.5 \pm 0.2 \\
2.5 \pm 0.2 \\
2.5 \pm 0.2\end{array}$ & $\begin{array}{l}0.2 \pm 0.0 \\
0.2 \pm 0.0 \\
1.6 \pm 0.1 \\
1.2 \pm 0.1 \\
1.0 \pm 0.1 \\
0.2 \pm 0.0\end{array}$ & $\begin{array}{r}110 \\
490 \\
1300 \\
1600 \\
1500 \\
240\end{array}$ & $\begin{array}{r}40 \\
180 \\
470 \\
610 \\
560 \\
90\end{array}$ \\
\hline SW extension & $-0.5,-0.9$ & $\begin{array}{l}0.6 \pm 0.1 \\
0.2 \pm 0.0\end{array}$ & $\begin{array}{l}-60.1 \pm 0.1 \\
-63.2 \pm 0.1\end{array}$ & $\begin{array}{l}1.8 \pm 0.1 \\
2.1 \pm 0.0\end{array}$ & $\begin{array}{l}\ldots \\
\ldots\end{array}$ & $\begin{array}{l}\ldots \\
\ldots\end{array}$ & $\begin{array}{l}\ldots \\
\ldots\end{array}$ \\
\hline \multicolumn{8}{|c|}{$\mathrm{CO}(2-1)$ with a beam area $0 . \prime 8 \times 0.0^{\prime \prime} 6$} \\
\hline IRS 1 & $+0.0,+0.0$ & $\begin{array}{r}-1.7 \pm 0.2 \\
-2.5 \pm 0.4 \\
-2.7 \pm 0.4 \\
-3.2 \pm 0.3 \\
1.3 \pm 0.2 \\
\end{array}$ & $\begin{array}{l}-63.7 \pm 0.3 \\
-59.0 \pm 0.3 \\
-57.0 \pm 0.3 \\
-54.0 \pm 0.3 \\
-48.2 \pm 0.3\end{array}$ & $\begin{array}{l}7.2 \pm 0.5 \\
2.0 \pm 0.2 \\
2.8 \pm 0.2 \\
3.2 \pm 0.3 \\
3.5 \pm 0.2\end{array}$ & $\begin{array}{c}0.7 \pm 0.2 \\
1.5_{-0.5}^{+0.7} \\
2.0_{-0.8}^{+1.0} \\
4.1_{-1.9}^{>5.0} \\
\ldots \\
\end{array}$ & $\begin{array}{l}\ldots \\
\ldots \\
\ldots \\
\ldots \\
\cdots\end{array}$ & $\begin{array}{l}\cdots \\
\cdots \\
\cdots \\
\cdots \\
\cdots \\
\end{array}$ \\
\hline \multicolumn{8}{|c|}{$\mathrm{HCN}(1-0)$ with a beam area $0^{\prime \prime} .8 \times 0^{\prime \prime} .7$} \\
\hline IRS 1 & $+0.0,+0.0$ & $\begin{array}{c}-0.7 \pm 0.1 \\
-0.8 \pm 0.1 \\
-0.3 \\
-0.1 \\
-0.7 \pm 0.1 \\
-0.2 \\
-0.1 \\
-0.6 \pm 0.1 \\
-0.2 \\
-0.4 \\
-1.1 \pm 0.1 \\
-1.0 \pm 0.1\end{array}$ & $\begin{array}{c}\ldots \\
-63.7 \pm 0.3 \\
\ldots \\
\ldots \\
-59.0 \pm 0.3 \\
\ldots \\
\ldots \\
-57.0 \pm 0.3 \\
\ldots \\
\ldots \\
-54.0 \pm 0.3 \\
\ldots\end{array}$ & $\begin{array}{c}7.1 \\
7.1 \pm 0.6 \\
7.1 \\
2.0 \\
2.0 \pm 0.1 \\
2.0 \\
2.8 \\
2.8 \pm 0.2 \\
2.8 \\
5.2 \\
5.2 \pm 0.3 \\
5.2\end{array}$ & $\begin{array}{c}\ldots \\
1.3_{-0.3}^{+0.5} \\
\ldots \\
\ldots \\
1.1_{-0.2}^{+0.4} \\
\ldots \\
\ldots \\
0.8_{-0.2}^{+0.2} \\
\ldots \\
\ldots \\
1.8_{-0.4}^{+0.7} \\
\ldots\end{array}$ & $\begin{array}{l}\ldots \\
\ldots \\
\ldots \\
\ldots \\
\ldots \\
\ldots \\
\ldots \\
\ldots \\
\ldots \\
\ldots \\
\ldots \\
\ldots\end{array}$ & $\begin{array}{l}\ldots \\
\ldots \\
\ldots \\
\ldots \\
\ldots \\
\ldots \\
\ldots \\
\ldots \\
\ldots \\
\ldots \\
\ldots \\
\ldots\end{array}$ \\
\hline
\end{tabular}

Notes. The reference position is R.A. $(\mathrm{J} 2000)=23^{\mathrm{h}} 13^{\mathrm{m}} 45^{\mathrm{s}} \cdot 37$, decl. $(\mathrm{J} 2000)=61^{\circ} 28^{\prime} 10^{\prime \prime} 43$.

${ }^{\mathrm{a}}$ With $T_{\mathrm{ex}}=200 \mathrm{~K}$.

${ }^{\mathrm{b}}$ With $T_{\mathrm{ex}}=75 \mathrm{~K}$.

We also extracted a ${ }^{13} \mathrm{CO}(2-1)$ spectrum toward the "SW tail" structure observed in the $1.3 \mathrm{~mm}$ continuum (Figure 13(b)). Toward the position of the SW extension in $1.3 \mathrm{~mm}$ continuum, a narrow $\left(\Delta V_{\mathrm{FWHM}} \approx 1.8 \mathrm{~km} \mathrm{~s}^{-1}\right)$ emission feature at $-60.1 \mathrm{~km} \mathrm{~s}^{-1}$ with a $\Delta S_{\mathrm{L}}=0.6 \mathrm{Jy}$ beam $^{-1}$ is seen close to $V_{\text {sys }}$, which seems to be affected by contamination from the spectrum from IRS 1 and has a skewed line profile. In addition, a narrow, weaker emission feature $\left(\Delta V_{\mathrm{FWHM}} \sim 2.1 \mathrm{~km} \mathrm{~s}^{-1}\right.$, $\Delta S_{\mathrm{L}}=0.2 \mathrm{Jy}$ beam $\left.^{-1}\right)$ at $-63.2 \mathrm{~km} \mathrm{~s}^{-1}$ is detected, which could be associated with low-velocity outflow gas.

\subsection{2. $C O(2-1)$ toward IRS 1}

We made channel maps of $\mathrm{CO}(2-1)$ (Figure 14), showing a wide absorption toward IRS 1 from -69 to $-52 \mathrm{~km} \mathrm{~s}^{-1}$. Multiple spectral components are included in this wide absorption, which are best seen in the $\mathrm{CO}(2-1)$ spectra toward the $1.3 \mathrm{~mm}$ emission peak in IRS 1 (Figure 13(c)) and the Gaussian fitting. Absorption features at velocities -63.7, -59.0, -57.0, and $-54.0 \mathrm{~km} \mathrm{~s}^{-1}$; an emission feature at $-48.2 \mathrm{~km} \mathrm{~s}^{-1}$; and the fitting results are summarized in Table 5 . The fitting results are in good agreement with ${ }^{13} \mathrm{CO}(2-1)$ except for the absorption feature at $-63.7 \mathrm{~km} \mathrm{~s}^{-1}$, which is much deeper $\left(\Delta S_{\mathrm{L}}=-1.7 \mathrm{Jy}\right)$ and much broader $\left(\Delta V_{\mathrm{FWHM}}=7 \mathrm{~km} \mathrm{~s}^{-1}\right)$ than its ${ }^{13} \mathrm{CO}(2-1)$ counterpart. The $\mathrm{CO}(2-1)$ counterpart corresponding to the blueshifted emission observed in ${ }^{13} \mathrm{CO}(2-1)$ at $-63.0 \mathrm{~km} \mathrm{~s}^{-1}$ was not detected, which might be because it is diluted by the strong and broad absorption feature at $-63.7 \mathrm{~km} \mathrm{~s}^{-1}$. The wide blueshifted $\mathrm{CO}(2-1)$ absorption is likely due to foreground outflow gas. The optical depths of the $\mathrm{CO}(2-1)$ features are determined with $\tau_{\mathrm{L}}=-\ln \left(1-\Delta S_{\mathrm{L}} / S_{\mathrm{C}}\right)$, showing the large optical depths $(\sim 3)$ of the $\mathrm{CO}(2-1)$ components that are associated with the redshifted absorption gas, and suggesting that the infalling $\mathrm{CO}(2-1)$ gas is optically thick.

\subsubsection{HCN(1-0) Hyperfine Lines and Absorption Profile}

The $J=1-0$ transition of $\mathrm{HCN}$ has three hyperfine lines $(F=0-1,1-1$, and $2-1)$ with optical depths in the ratio 1:3:5 under LTE. The strongest line $F=2-1$ has $v_{0}=88.6318 \mathrm{GHz}$. The velocity separations of $F=1-1$ and $F=0-1$ with respect to $F=2-1$ are +4.84 and $-7.08 \mathrm{~km} \mathrm{~s}^{-1}$, respectively. We made multiple-Gaussian fits to the four kinematic components in absorption identified in $\mathrm{CO}(2-1)$ to the $\mathrm{HCN}(1-0)$ absorption profile (Figure 13(d)). For each kinematic component, we used the three hyperfine lines of the $\mathrm{HCN}(1-0)$ transition in the initial fitting. In the fits, the intensity ratio of each hyperfine line was fixed assuming LTE, and the FWHM line widths for the hyperfine components were the same for each kinematic component. The three parameters $\Delta S_{\mathrm{L}}, V_{\mathrm{LSR}}$, and $\Delta V_{\mathrm{FWHM}}$ for the main hyperfine lines $(F=2-1)$ were determined from the best fitting. However, the hyperfine lines $F=0-1$ and 

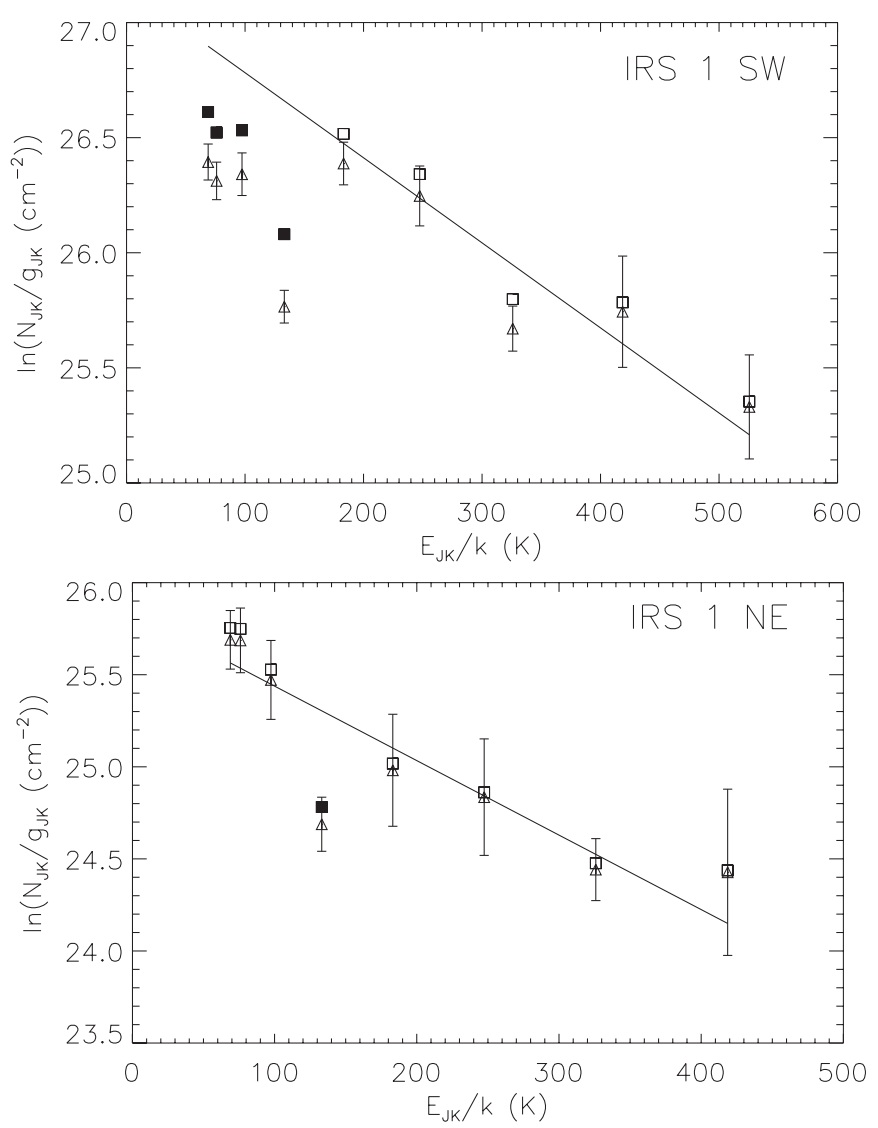

Figure 10. Population diagrams with $K=0,1,2,4,5,6,7$, and 8 transitions of $\mathrm{CH}_{3} \mathrm{CN}(12-11)$ lines for IRS $1 \mathrm{SW}$, and $K=0,1,2,4,5,6$, and 7 transitions for NE. The vertical bar marks $1 \sigma$ error. The corresponding measurement of $K=3$ transition is marked with filled a square, but excluded from the fitting. The straight lines are the best fits to the data based on linear regression for $\ln \left(N_{J K} / g_{J K}\right)=\alpha\left(E_{J K} / k\right)+\beta$, with $\alpha=-0.0038 \pm 0.0004$ and $\beta=27.5 \pm 0.1$ for IRS $1 \mathrm{SW}$ and $\alpha=-0.0038 \pm 0.0012$ and $\beta=25.8 \pm 0.4$ for NE.

$F=1-1$ in the high blueshifted and high redshifted ends of the absorption profile require high intensities that do not fit to their corresponding LTE ratio. The high-velocity HCN line features might be non-LTE. Alternatively, the broadening of the $\mathrm{HCN}(1-0)$ absorption profile might be caused by highvelocity gas components, which are associated with shocked or compressed gas and traced by the HCN emission (e.g., Shi et al. 2010). The absorptions caused by these high-velocity gas components might be insignificant in $\mathrm{CO}(2-1)$ due to the mitigation of the emission of the $\mathrm{CO}(2-1)$ outflow. Table 5 summarizes the fitting results.

\section{DISCUSSION}

\subsection{A Massive Ionizing Star in the HC H II Region IRS 1}

IRS 1 is a $\mathrm{HC} \mathrm{H}$ II region associated with a newborn star. At the high-angular resolution $\left(\sim 00^{\prime} 3\right)$ in the CARMA observations, the surrounding dust emission has been resolved out. Fitting the continuum fluxes of IRS 1 at multiple wavelengths indicates that even at $1.3 \mathrm{~mm}$ the free-free emission still dominates the continuum emission (Sandell et al. 2009). If we assume optically thin emission and neglect the contribution of the thermal dust emission, a Lyman continuum flux $N_{\text {Lym }}$ can be estimated from the $1.3 \mathrm{~mm}$ flux density $\left(S_{\mathrm{C}}=3.8 \mathrm{Jy}\right.$ at $\left.v=224 \mathrm{GHz}\right)$ of IRS 1 with the equation below of a recombination coefficient of $\alpha_{\mathrm{B}}=2.58 \times 10^{-13} \mathrm{~cm}^{-3} \mathrm{~s}^{-1}$ in case $\mathrm{B}$ and electron temperature of $T_{\mathrm{e}}=1 \times 10^{4} \mathrm{~K}$ (Hummer \& Seaton 1963),

$$
\begin{aligned}
N_{\text {Lym }}= & 7.6 \times 10^{46} \text { photons s }{ }^{-1} \alpha^{-1}\left(v, T_{\mathrm{e}}\right) \\
& \times\left(\frac{T_{\mathrm{e}}}{10^{4} \mathrm{~K}}\right)^{0.35}\left(\frac{v}{\mathrm{GHz}}\right)^{0.1}\left(\frac{S_{\mathrm{C}}}{\mathrm{Jy}}\right)\left(\frac{D}{\mathrm{kpc}}\right)^{2},
\end{aligned}
$$

where the correction factor for power-law approximation $\alpha\left(\nu, T_{\mathrm{e}}\right)=0.85$ for $T_{\mathrm{e}}=10,000 \mathrm{~K}$ and $\nu=224 \mathrm{GHz}$ (Mezger \& Henderson 1967). For $D=2.65 \mathrm{kpc}$, we have $N_{\text {Lym }}=4.1 \times 10^{48}$ photons s $^{-1}$. At $224 \mathrm{GHz}$, our observations may not be sensitive enough to reveal the extended structures of the ionized outflow as the VLA observations show (see Figure 2). However, with Equation (2) and the $U$-band measurement (60 mJy; see Sandell et al. 2009), it suggests that only $\sim 2 \times 10^{46}$ photons $\mathrm{s}^{-1}$ were contributed by the extended structure, less than $1 \%$ of the $224 \mathrm{GHz}$ results.

There are a few caveats that need to be kept in mind for the above estimate. First, most of the free-free emission in the unresolved central region $(\leqslant 0$.'1) is actually optically thick, resulting in an underestimation of the $u v$ photon flux from the young stellar object (YSO) in IRS 1. Second, the contribution of dust thermal emission may not be negligible. If we extrapolate the total flux-density fit for IRS 1 from the spectrum between 4.8 and $43.4 \mathrm{GHz}$ (Sandell et al. 2009) to $224 \mathrm{GHz}$, a total flux of $\sim 2.6 \mathrm{Jy}$ from free-free emission is predicted, implying that dust thermal emission might contribute $\sim 30 \%$ of the total flux density. In addition, ionization is not only due to the UV photons from the stellar photosphere, but also caused by the heating in the accretion process. For low-mass pre-main-sequence stars (classical $\mathrm{T}$ Tauri stars), the shock front due to accretion will produce hot plasma with a temperature of several $10^{6} \mathrm{~K}$ or $10^{6}-10^{7} \mathrm{~K}$ and yield significant emission in the soft X-ray band (Argiroffi et al. 2012). It is possible that in the case of high-mass YSOs with ongoing accretion similar processes also occur, and produce high-energy photons that will ionize surrounding molecular gas. Unfortunately, there is currently no detailed quantitative model on how much Lyman continuum flux the accretion process can contribute for the high-mass YSOs. However, we believe that the $u v$ photons from the stellar photospheres of the YSOs are the dominating source for photoionization.

The massive ionizing star should be the primary energy source, responsible for ionizing the $\mathrm{HC} \mathrm{H}$ II region and driving the energetic ionized outflow via the overwhelming radiative pressure. Neglecting all the factors described above, the Lyman continuum flux of $\sim 4.1 \times 10^{48}$ photons $\mathrm{s}^{-1}$ is equivalent to the flux from a single star of O8-O9.5 in the main sequence (Vacca et al. 1996; Martins et al. 2005), which is required to maintain the ionization of the $\mathrm{HC} \mathrm{H}$ II region IRS 1 alone. Taking into account the possible overestimation of $30 \%$ due to dust thermal emission will not change the derived spectral type. Considering the fact that we may have significantly underestimated the continuum flux due to optical-depth effects, the assessment for the spectral type of the ionizing star is also consistent with the suggestions of an O6/7 star estimated previously (e.g., Willner 1976; Akabane \& Kuno 2005) if the turn-over frequency for the free-free emission from the $\mathrm{HC} \mathrm{H}$ II region is significantly higher than $230 \mathrm{GHz}(\lambda \sim 1.3 \mathrm{~mm})$.

\subsection{Infalling Gas}

As suggested by Corder (2008), Sandell et al. (2009), and Beuther et al. (2012), an ongoing accretion might be taking place in the IRS 1 system. From the ${ }^{13} \mathrm{CO}(2-1)$ absorption in 


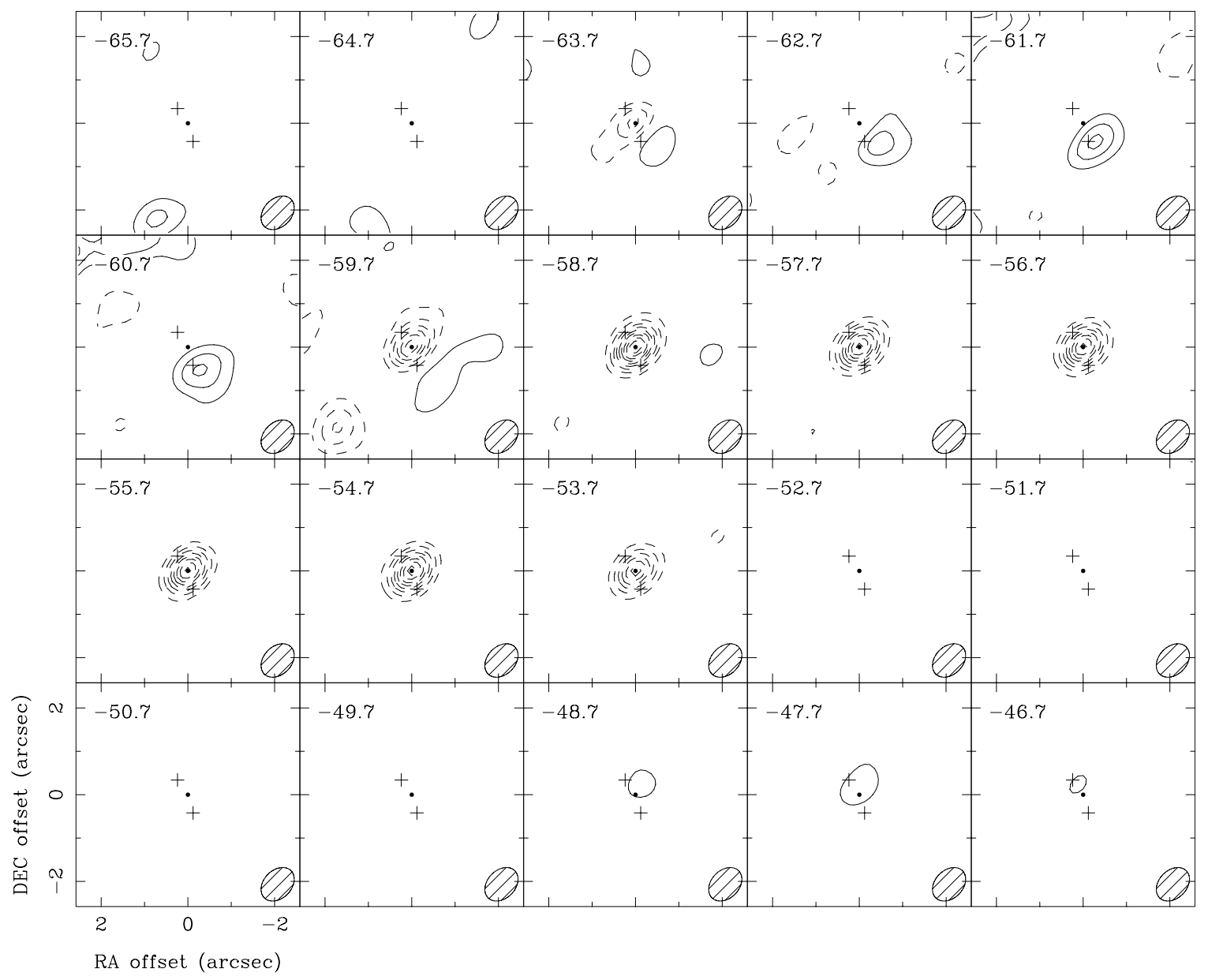

Figure 11. Channel maps of ${ }^{13} \mathrm{CO}(2-1)$. The contours are $(\ldots,-4,-3,-2,-1,1,2, \ldots) \times 5 \sigma$, and $1 \sigma=0.05 \mathrm{Jy}_{\text {beam }}^{-1}$. The FWHM beam size is $0{ }^{\prime \prime} 8 \times 0$ ' 6 , P.A. $=-44^{\circ}$. IRS 1 is located at the phase center (indicated by the filled black circle).

front of the IRS 1 core, the total column density of molecular gas is $2.0 \times 10^{24} \mathrm{~cm}^{-2}$ using $[\mathrm{CO}] /\left[\mathrm{H}_{2}\right]=10^{-4},\left[{ }^{13} \mathrm{CO}\right] /[\mathrm{CO}]=$ $1 / 60$, and a high $T_{\mathrm{ex}}=200 \mathrm{~K}$ (see Table 5). If the molecular gas is spherically and evenly distributed within a radius of $1900 \mathrm{AU}$ (equivalent to the 0!7 FWHM beam size of the CARMA $1.3 \mathrm{~mm}$ observations), this column density implies that about $5 M_{\odot}$ in the surrounding region is available for accretion onto IRS 1. On the other hand, if we adopt a large-scale distribution of the ${ }^{13} \mathrm{CO}$ gas (the lower column density of $8 \times 10^{23} \mathrm{~cm}^{-2}$ corresponding to the lower $T_{\mathrm{ex}}=75 \mathrm{~K}$ needs to be considered), the mass of the molecular gas associated with IRS 1 would be larger because its value is proportional to the square of radius.

The inverse P-Cygni profile revealed from the observations of $\mathrm{HCO}^{+}(1-0)$ by Corder (2008) and Sandell et al. (2009) suggests that the infall likely occurs within the beam size of 4 ". 5 , or the inner region with a size of $0.06 \mathrm{pc}$. Our subarcsecond resolution observations of $\mathrm{HCN}(1-0), \mathrm{CO}(2-1)$, and ${ }^{13} \mathrm{CO}(2-1)$ lines toward IRS 1 agree with the previous results.

Absorption features are also detected in OCS(19-18) and $K=$ 2-5 components of $\mathrm{CH}_{3} \mathrm{CN}(12-11)$ (see Sections 4.3 and 4.4) in the velocity range between -56.5 and $-53.0 \mathrm{~km} \mathrm{~s}^{-1}$, corresponding to the absorption features at -57.0 and $-54.0 \mathrm{~km} \mathrm{~s}^{-1}$ in $\mathrm{HCN}(1-0), \mathrm{CO}(2-1)$, and ${ }^{13} \mathrm{CO}(2-1)$. These absorptions probably arise in the infalling gas, revealing the hot dense gas in the accretion flow. The hot molecular gas is more redshifted than the corresponding $\mathrm{HCN}(1-0)$ and $\mathrm{CO}$ absorptions, perhaps tracing an accelerating infall closer to IRS 1.
The redshifted ${ }^{13} \mathrm{CO}(2-1)$ absorption against the continuum radiation from IRS 1 clearly shows evidence for ongoing gas infall. From the optical depth of the infalling gas determined from the relatively optically thin line ${ }^{13} \mathrm{CO}(2-1)$, as well as the excitation temperature determined from the $\mathrm{CH}_{3} \mathrm{CN}$ population diagram, we can estimate the infall rate $d M / d t=$ $4 \pi r^{2} m_{\mathrm{H}} \mu n_{\mathrm{H}} V_{\mathrm{in}}$, at an infall radius $r$, where the mean molecular weight is $\mu=2.35$ and density of the accretion materials is $n_{\mathrm{H}}=N_{\mathrm{H}} / r$. Thus, the infall rate is a function of the measurable quantities,

$$
\begin{gathered}
\frac{d M}{d t}=2.8 \times 10^{-4} M_{\odot} \mathrm{yr}^{-1}\left[\frac{\theta_{\text {in }}}{\operatorname{arcsec}}\right]\left[\frac{D}{\mathrm{kpc}}\right] \\
{\left[\frac{N_{\mathrm{H}}}{10^{24} \mathrm{~cm}^{-2}}\right]\left[\frac{\Delta V_{\text {in }}}{\mathrm{km} \mathrm{s}^{-1}}\right],}
\end{gathered}
$$

where the angular size of the infall region $\theta_{\text {in }}$ is approximately the geometric mean of the telescope beam $\theta_{\mathrm{B}}=0^{\prime \prime} .7$.

The two redshifted absorption components at -54.5 and $-57.0 \mathrm{~km} \mathrm{~s}^{-1}$ in ${ }^{13} \mathrm{CO}(2-1)$ give $\mathrm{H}_{2}$ column densities $\left(N_{\mathrm{H}, \mathrm{red}}\right)$ of $9 \times 10^{23}$ and $1 \times 10^{24} \mathrm{~cm}^{-2}$, respectively. If we take the velocity displacements of the two absorption features from the systemic velocity as their corresponding infall velocity $\left(V_{\text {in }} \approx \Delta V=V_{\mathrm{LSR}}-V_{\mathrm{sys}}\right)$, an infall rate of $3 \times 10^{-3} M_{\odot} \mathrm{yr}^{-1}$ can be inferred based on Equation (3) for the IRS 1 region, higher than the value derived by previous studies (Corder 


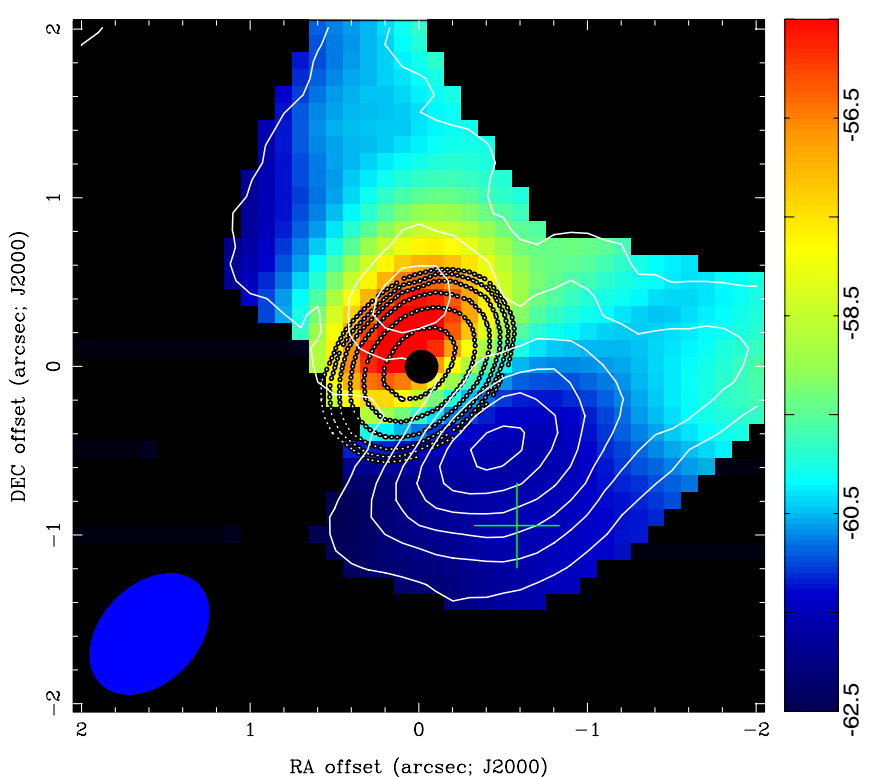

Figure 12. Images of ${ }^{13} \mathrm{CO}(2-1)$ emission (solid white contours) and absorption (dotted contours) constructed by integrating the line intensity from the velocity range between -43 and $-66 \mathrm{~km} \mathrm{~s}^{-1}$ with an FWHM beam size $0{ }^{\prime \prime} 8 \times$ 0.6 (P.A. $=-45^{\circ}$ ). The emission contours are 2, 2.5, 3, 3.5, 4, and $4.5 \mathrm{Jy}$ beam $^{-1} \mathrm{~km} \mathrm{~s}^{-1}$. The absorption contours are $-2,-2.8,-4,-5.7,-8$, and-11.3 Jy beam ${ }^{-1} \mathrm{~km} \mathrm{~s}^{-1}$. The intensity-weighted radial velocity (LSR) image (color) was made from the ${ }^{13} \mathrm{CO}(2-1)$ emission line in the same velocity range used for the total intensity image of the line emission. The color wedge scales the velocities in the units of $\mathrm{km} \mathrm{s}^{-1}$. The green plus sign indicates the emission peak of the SW $1.3 \mathrm{~mm}$ continuum extension.

(A color version of this figure is available in the online journal.)
2008; Sandell et al. 2009), lower than that of Beuther et al. (2012), and in agreement with that of Qiu et al. (2011). In addition, as discussed in Section 4.6.1, we might underestimate the size of the infalling region, and thus overestimate the characteristic excitation temperature of the ${ }^{13} \mathrm{CO}$ gas in this region. However, because the column density $N_{\mathrm{H}}$ in Equation (3) is proportional to excitation temperature, we expect that the different approach (larger infalling region, low characteristic $T_{\mathrm{ex}}$ ) would not significantly change the derived infall rate.

The infall rate can also be estimated from the $\mathrm{HCN}(1-0)$ results despite larger uncertainty in the line profile fitting and the poor determination of the abundance. On the basis of Rolfs \& Wilson (2004), the column density of HCN can be derived by:

$$
N(\mathrm{HCN})=5.41 \times 10^{11} \mathrm{~cm}^{-2} \frac{T_{\mathrm{ex}} \int \tau_{v} \mathrm{~d} v}{1-\exp \left(-4.25 / T_{\mathrm{ex}}\right)} .
$$

With $T_{\mathrm{ex}}=200 \mathrm{~K}$, the two redshifted absorption $\mathrm{HCN}(1-0)$ components at -54.0 and $-57.0 \mathrm{~km} \mathrm{~s}^{-1}$ give $\mathrm{HCN}$ column densities of $N_{\mathrm{HCN}}=5 \times 10^{16}$ and $N_{\mathrm{HCN}}=1 \times 10^{16} \mathrm{~cm}^{-2}$, respectively. A recent study reported a $\mathrm{H}^{13} \mathrm{CN}$ abundance of $\sim 2 \times 10^{-10}$ in active star formation regions (Zinchenko et al. 2009), so we estimate a total $\mathrm{H}_{2}$ column density $N\left(\mathrm{H}_{2}\right)=5 \times$ $10^{24} \mathrm{~cm}^{-2}$ with $\left[\mathrm{H}^{13} \mathrm{CN}\right] /[\mathrm{HCN}]=1 / 60$, which corresponds to an infall rate of $1 \times 10^{-2} M_{\odot} \mathrm{yr}^{-1}$, triple the value derived from ${ }^{13} \mathrm{CO}(2-1)$. Although the infall rates derived from ${ }^{13} \mathrm{CO}$ $(2-1)$ and $\mathrm{HCN}(1-0)$ are relatively high, they are still in the typical range of $10^{-4}-10^{-2} M_{\odot} \mathrm{yr}^{-1}$ for embedded high-mass cores (Wu et al. 2009, and the references therein).

We can compare the derived infall rates with an ideal free-fall case. With an enclosed mass of $30 M_{\odot}$ (including the mass of
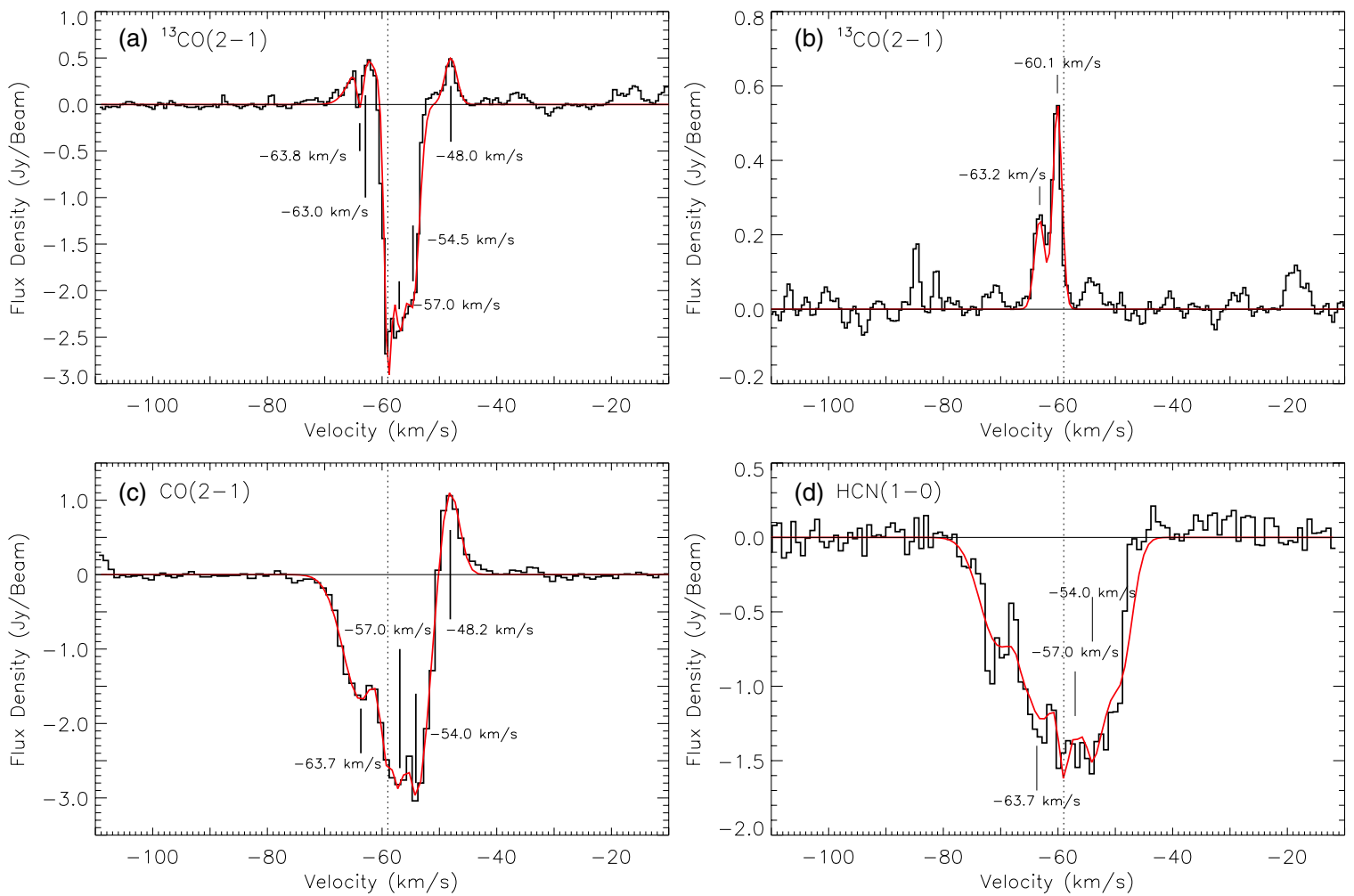

Figure 13. (a), (c), and (d) The ${ }^{13} \mathrm{CO}(2-1), \mathrm{CO}(2-1)$, and $\mathrm{HCN}(1-0)$ spectra toward IRS 1, respectively. (b) The ${ }^{13} \mathrm{CO}(2-1)$ spectrum toward the position of the $1.3 \mathrm{~mm} \mathrm{SW}$ extension. In all the figures, the dashed vertical lines mark $V_{\text {sys }}=-59.0 \mathrm{~km} \mathrm{~s}^{-1}$, and the red curves are the multiple-Gaussian fitting to the line profiles. The parameters for each of the kinematic components from the fitting are summarized in Table 5.

(A color version of this figure is available in the online journal.) 


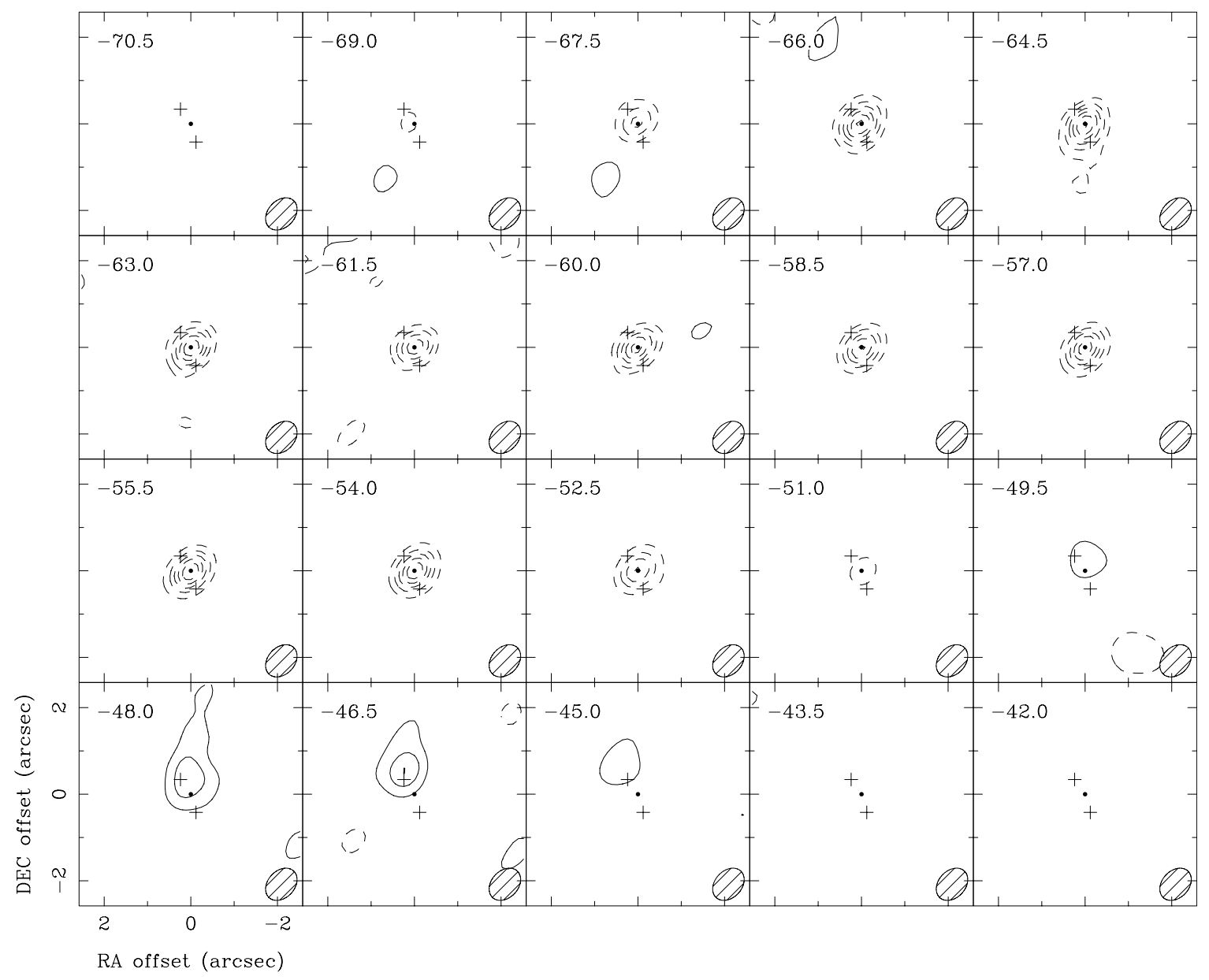

Figure 14. Channel maps of $\mathrm{CO}(2-1)$. The contours are $(\ldots,-4,-3,-2,-1,1,2,3,4, \ldots) \times 5 \sigma$, and $1 \sigma=0.1 \mathrm{Jy}^{\text {beam }}{ }^{-1}$. The FWHM beam size is 0 ' $8 \times 0$ ' 6 , P.A. $=-42^{\circ}$. IRS 1 is located at the phase center (indicated by the filled black circle).

both gas and the central star) at a radius $r=0.7$, the free-fall infall velocity $V_{\text {in,ff }}$ is about $5.5 \mathrm{~km} \mathrm{~s}^{-1}$, corresponding to a freefall infall rate of $5 \times 10^{-3} M_{\odot} \mathrm{yr}^{-1}$ from the ${ }^{13} \mathrm{CO}(2-1)$ results. This value agrees with $3 \times 10^{-3} M_{\odot} \mathrm{yr}^{-1}$, calculated from the velocity displacements of the redshifted ${ }^{13} \mathrm{CO}(2-1)$ absorption features, indicating that gas is falling into IRS 1 rapidly.

In addition to the redshifted absorption velocity components, we see a blueshifted absorption at $V_{\mathrm{lsr}}=-63.8 \mathrm{~km} \mathrm{~s}^{-1}$ in ${ }^{13} \mathrm{CO}(2-1)$ or $V_{\mathrm{lsr}}=-63.7 \mathrm{~km} \mathrm{~s}^{-1}$ in $\mathrm{CO}(2-1)$ and $\mathrm{HCN}(1-0)$. Since the optical depths of the blueshifted absorption are significantly lower than the redshifted ones, and no other observations have suggested a cloud component in NGC 7538 region at this radial velocity that is only seen toward IRS 1 , we believe that the absorption line components at -63.7 or $-63.8 \mathrm{~km} \mathrm{~s}^{-1}$ arise from the blueshifted outflow gas between IRS 1 and the observer.

\subsection{The Nature of Hot Molecular Clumps and Dynamics in NGC 7538 IRS 1}

The kinematics observed from IRS $1 \mathrm{SW}$ and NE in the central $2^{\prime \prime}$, from OCS(19-18), $\mathrm{CH}_{3} \mathrm{CN}(12-11)$, and ${ }^{13} \mathrm{CO}(2-1)$ show a velocity gradient in P.A. $\sim 43^{\circ}$. The three molecular species observed in this paper are in good agreement with the results from the ${ }^{13} \mathrm{CO}(1-0)$ emission (Scoville et al. 1986) and ${ }^{15} \mathrm{NH}_{3}$ masers (Gaume et al. 1991) observations.

The observed high excitation temperatures and velocities of the hot molecular components in the circumstellar region of
IRS 1 provide clues for understanding the IRS 1 system. IR radiation from IRS 1 supplies the primary heating in the central $1^{\prime \prime}-2^{\prime \prime},(\sim 5000 \mathrm{AU})$. The ionized emission observed with the VLA in the inner $<2^{\prime \prime}$ (Campbell 1984; Gaume et al. 1995; Sandell et al. 2009) is suggested to be a hot ionized wind with a velocity up to $300 \mathrm{~km} \mathrm{~s}^{-1}$, which is partially confined and collimated by the molecular material surrounding IRS 1 (Gaume et al. 1995). The hot molecular components (see Figures 6(a) and 9(a)) are located NE of the ionized core, and SW of the southern lobe of the ionized outflow. These two locations are close to the path of the ionized outflow, suggesting that the molecular gas may be heated by the interaction between the ionized outflow and accreting gas.

A large accretion flow with a rate of $3-10 \times 10^{-3} M_{\odot} \mathrm{yr}^{-1}$ continues to accumulate matter into the circumstellar region around IRS 1 (see Section 5.2). Confronting the overwhelming pressure from the accretion flow, the $\mathrm{HC} \mathrm{H}$ II region surrounding the $\mathrm{O}$ star must be highly confined and difficult to expand to form a larger size $\mathrm{H}$ II region. The excess angular momentum in the infalling gas must be transported out. The outflow provides a mechanism for transferring the angular momentum of the infalling gas.

The large-scale $\mathrm{CO}(2-1),{ }^{13} \mathrm{CO}(1-0)$, and $\mathrm{HCO}^{+}(1-0)$ images from CARMA, SMA, and single-dish observations suggest a bipolar outflow in a north-south direction with an opening angle greater than $\sim 90^{\circ}$. The interaction between this wideangle molecular outflow and the envelope of ambient molecular 
gas around IRS 1 might excite the observed hot molecular components, redshifted to the NE and blueshifted to the SW. The density of the environment is much higher to the south of IRS 1; thus the molecular emission from IRS $1 \mathrm{SW}$ is stronger, and the ionized outflow in this direction is highly confined.

The redshifted radial velocity of IRS 1 NE could also be due to rotation of the wide-angle outflow around its axis. An ionized outflow that rotates around its axis could entrain the adjacent molecular gas, and consequently, the impact of the ionized outflow on the circumstellar gas could produce highly excited molecular species at the positions of IRS 1 NE and SW.

The ambient density is higher to the south and SW of IRS 1, so the entrainment of the ionized jet is not significant. To the north of IRS 1 , the density is lower, and the ionized outflow can penetrate and produce significant redshifted movement of the entrained gas NE of IRS 1 . We can roughly estimate the angular momentum of the NE component to be $3.6 \times 10^{17} M_{\odot}$ $\mathrm{m}^{2} \mathrm{~s}^{-1}$ by assuming a mass $M_{\mathrm{NE}}=1 M_{\odot}$ (see Section 4.5$)$, rotation velocity $v_{\mathrm{NE}}=8 \mathrm{~km} \mathrm{~s}^{-1}$, and radius $r_{\mathrm{NE}}=300 \mathrm{AU}$ $\left(\Delta \alpha=0^{\prime \prime} .11\right.$, see Table 4). The angular momentum input to IRS 1 is about $5.7 \times 10^{15} M_{\odot} \mathrm{m}^{2} \mathrm{~s}^{-1} \mathrm{yr}^{-1}$ with an infall rate of $5 \times 10^{-3} M_{\odot} \mathrm{yr}^{-1}$, a radius of $1900 \mathrm{AU}\left(\sim 0^{\prime \prime} .7\right)$, and an enclosed mass of $30 M_{\odot}$. Therefore, it is plausible that the ionized outflow could cause the observed redshifted movement of the component $\mathrm{NE}$ in the lifetime of the accretion process.

\section{CONCLUSION}

Using subarcsecond resolution observations at 1.3 and $3.4 \mathrm{~mm}$ with SMA and CARMA, we analyzed the molecular lines and continuum emission from NGC 7538 IRS 1 to investigate the dynamic processes related to the star-forming activities in this region. With an image convolved to 0 '. 1 from CARMA $\mathrm{B}$-array observation at $1.3 \mathrm{~mm}$, the primary continuum source IRS 1, an HC HII region, was resolved into two components, an unresolved compact core with a linear size $<270 \mathrm{AU}$ and a north-south extended lobe of the ionized outflow in agreement with the predicted extrapolation of a power-law dependence made by Sandell et al. (2009). Both the SMA and CARMA show extended dust continuum emission to the SW of IRS 1, which may indicate a reservoir of molecular gas.

The molecular lines $\mathrm{OCS}(19-18), \mathrm{CH}_{3} \mathrm{CN}(12-11)$, and ${ }^{13} \mathrm{CO}(2-1)$ show that dense molecular gas is primarily distributed southwest to northeast across IRS 1 with an angular size of $\sim 2^{\prime \prime} \times 1^{\prime \prime}$. The locations and velocities of the molecular clumps suggest that they may be heated by interaction between the ionized outflow from IRS 1 and the adjacent molecular gas in a rotating wide-angle outflow in a $\mathrm{N}-\mathrm{S}$ direction from IRS 1.

From high-resolution $\left(0^{\prime} .8 \times 00^{\prime \prime} 6\right)$ observations of ${ }^{13} \mathrm{CO}(2-1)$, $\mathrm{CO}(2-1)$, and $\mathrm{HCN}(1-0)$, we fitted multiple-Gaussian velocity components to the spectra toward the continuum sources in IRS 1 . We found that the significant molecular lines in absorption are redshifted with respect to the systemic velocity toward IRS 1, suggesting that a large amount of material surrounding the region is infalling toward IRS 1 . An infall rate of $\sim 3-10 \times$ $10^{-3} M_{\odot} \mathrm{yr}^{-1}$ was inferred from ${ }^{13} \mathrm{CO}(2-1)$ and $\mathrm{HCN}(1-0)$ lines.

L.Z. is supported by National Basic Research Program of China (973 program) No. 2012CB821802. He was a SAO predoctoral fellow, and a part of the work in this paper was carried out during the course of his $\mathrm{PhD}$ research. J.H.Z. is grateful to the National Astronomical Observatories of China for hosting his visit during the course of writing this research paper. We are grateful to Miller Goss for his helpful comments and suggestions. Support for CARMA construction was derived from the states of California, Illinois, and Maryland, the Gordon and Betty Moore Foundation, the Kenneth T. and Eileen L. Norris Foundation, the Associates of the California Institute of Technology, and the National Science Foundation. Ongoing CARMA development and operations are supported by the National Science Foundation under a cooperative agreement, and by the CARMA partner universities. The Very Large Array (VLA) is operated by the National Radio Astronomy Observatory (NRAO). The NRAO is a facility of the National Science Foundation operated under cooperative agreement by Associated Universities, Inc.

\section{REFERENCES}

Akabane, K., \& Kuno, N. 2005, A\&A, 431, 183

Araya, E., Hofner, P., Kurtz, S., Bronfman, L., \& Dedeo, S. 2005, ApJS, 157,279

Argiroffi, C., Maggio, A., Montmerle, T., et al. 2012, ApJ, 752, 100

Beuther, H., Linz, H., \& Henning, T. 2012, A\&A, 543, 88

Brogan, C. L., Hunter, T. R., Indebetouw, R., et al. 2008, Ap\&SS, 313, 53

Campbell, B. 1984, ApJL, 282, L27

Churchwell, E., Walmsley, C. M., \& Wood, D. O. S. 1992, A\&A, 253, 541

Corder, S. 2008, PhD thesis, Caltech

Davis, C. J., Moriarty-Schieven, G., Eislöffel, J., Hoare, M. G., \& Ray, T. P. 1998, AJ, 115, 1118

De Buizer, J. M., \& Minier, V. 2005, ApJL, 628, L151

Furuya, R. S., Cesaroni, R., Takahashi, S., et al. 2008, ApJ, 673, 363

Gaume, R. A., Goss, W. M., Dickel, H. R., Wilson, T. L., \& Johnston, K. J. 1995, ApJ, 438, 776

Gaume, R. A., Johnston, K. J., Nguyen, H. A., et al. 1991, ApJ, 376, 608

Goldsmith, P. F., \& Langer, W. D. 1999, ApJ, 517, 209

Hollis, J. M. 1982, ApJ, 260, 159

Hummer, D. G., \& Seaton, M. J. 1963, MNRAS, 125, 437

Kameya, O., Hasegawa, T. I., Hirano, N., Takakubo, K., \& Seki, M. 1989, ApJ, 339,222

Klaassen, P. D., Wilson, C. D., Keto, E. R., \& Zhang, Q. 2009, ApJ, 703, 1308

Knez, C., Lacy, J. H., Evans, N. J., II, van Dishoeck, E. F., \& Richter, M. J. 2009, ApJ, 696, 471

Kraus, S., Balega, Y., Elitzur, M., et al. 2006, A\&A, 445, 521

Loren, R. B., \& Mundy, L. G. 1984, ApJ, 286, 232

Martins, F., Schaerer, D., \& Hillier, D. J. 2005, A\&A, 436, 1049

Mezger, P. G., \& Henderson, A. P. 1967, ApJ, 147, 471

Minier, V., Booth, R. S., \& Conway, J. E. 1998, A\&A, 336, L5

Minier, V., Booth, R. S., \& Conway, J. E. 2000, A\&A, 362, 1093

Moscadelli, L., Reid, M. J., Menten, K. M., et al. 2009, ApJ, 693, 406

Nummelin, A., Bergman, P., Hjalmarson, A., et al. 1998, ApJS, 117, 427

Pestalozzi, M. R., Elitzur, M., Conway, J. E., \& Booth, R. 2004, ApJL, 603, L113

Qin, S.-L., Zhao, J.-H., Moran, J. M., et al. 2008, ApJ, 677, 353

Qiu, K., Zhang, Q., \& Menten, K. M. 2011, ApJ, 728, 6

Rolfs, K., \& Wilson, T. L. (ed.) 2004, Tools of Radio Astronomy (Berlin: Springer)

Sandell, G., Goss, W. M., Wright, M., \& Corder, S. 2009, ApJL, 699, L31

Sandell, G., \& Sievers, A. 2004, ApJ, 600, 269

Sault, R. J., Teuben, P. J., \& Wright, M. C. H. 1995, in ASP Conf. Ser. 77, Astronomical Data Analysis Software and Systems IV, ed. R. A. Shaw, H. E. Payne, \& J. J. E. Hayes (San Francisco, CA: ASP), 433

Scoville, N. Z., Sargent, A. I., Sanders, D. B., et al. 1986, ApJ, 303, 416

Shi, H., Zhao, J.-H., \& Han, J. L. 2010, ApJL, 718, L181

Sutton, E. C., Blake, G. A., Masson, C. R., \& Phillips, T. G. 1985, ApJS, 58,341

Vacca, W. D., Garmany, C. D., \& Shull, J. M. 1996, ApJ, 460, 914

Willner, S. 1976, ApJ, 206, 728

Wu, Y., Qin, S.-L., Guan, X., et al. 2009, ApJL, 697, L116

Zinchenko, I., Caselli, P., \& Pirogov, L. 2009, MNRAS, 395, 2234 